\title{
PROTECTIVE EFFECT OF GRAPE SEEDS EXTRACT AGAINST SODIUM NITRITE-INDUCED TOXICITY AND OXIDATIVE STRESS IN ALBINO RATS
}

\author{
*Gihan, M. Hammoud, Nivin, S. Nail, Khaled, M. ELSawy, Adel,S. Abd \\ El-Rahman, Mervat, S.H. Youssef and Mohamed, S. Masoud \\ * Regional Center for Food and Feed (RCFF), Agricultural Research Center (ARC), \\ Giza, Egypt.
}

\begin{abstract}
Sodium nitrite $\left(\mathrm{NaNO}_{2}\right)$ is important antimicrobial, flavoring, coloring and preservative agent in meat and fish product. However, nitrite may cause methemoglobinemia and other illness, and may react with certain amines to form carcinogenic nitrosamines. The study aimed to evaluate the efficacy of grape seeds extract (GSE) as a potential novel and useful strategy for the modulation of oxidative stress and toxicity induced by $\mathrm{NaNo}_{2}$ in male rats. Sodium nitrite was used at two dose levels 1 and $2 \mathrm{~g} / \mathrm{L}$ and orally administrated to rats in drinking water for 4 and 8 weeks. Ingestion of $\mathrm{NaNO}_{2}$, resulted in significant time and dosedependent reduction in RBCs count, WBCs count, hemoglobin $(\mathrm{Hb})$ content, serum albumin and total protein (TP), plasma reduced glutathione (GSH), glutathione -S- transferase (GST), superoxide dismutase (SOD) contents in the treated rats. whereas, blood methemoglobin (MetHb), serum alanine transaminase (ALT), aspartate aminotransferase (AST), alkaline phosphatase (ALP), gamma glutamyl transpeptidase (GGT), creatinine, urea, triglycerides, total cholesterol and lipid peroxidation product (malondialdehyde, MDA) were significantly increased in dose and time dependant manner after implication of this preservative. Hyperglycemia was observed in $\mathrm{NaNO}_{2}$ ingested rats. Moreover, histopathological examination of $\mathrm{NaNO}_{2}$ treated rats at the end of experiment revealed marked alterations in liver, kidneys, brain, heart, lung and testes. The degree of severity of noted lesions increased with increase of administrated dose. Fortunately, synergistic administration of ethanolic GSE $\left(150 \mathrm{mg} / \mathrm{kg}\right.$ body weight (B.W)/day) and $\mathrm{NaNO}_{2}$ resulted in significant amelioration of negative effect of $\mathrm{NaNO}_{2}$ on the investigated biochemical parameters and improvement of investigated antioxidant especially with low $\mathrm{NaNO}_{2}$ dose and longer time. Moreover, GSE administration was able to protect most of tested organs against low $\mathrm{NaNO}_{2}$ dose and minimize the damage induced by high $\mathrm{NaNO}_{2}$ dose. It was concluded that GSE supplementation could be considered as a promising antioxidant in reducing oxidative stress and toxicity of $\mathrm{NaNO}_{2}$.
\end{abstract}

Key words: Sodium nitrite, Grape seeds extract, Oxidative stress, Biochemical parameters, and Pathological examination.

\section{INTRODUCTION}

Sodium nitrite $\left(\mathrm{NaNO}_{2}\right)$ is an inorganic salt used in the manufacture of dyes and considered as one of the important food additives for fishes and meat that has been used in vivo and in vitro experiments for decades to prevent growth of Clostridium botulinum, the bacteria which causes botulism (Luca et al., 1987). Also, it is used in drug industries and in medicine as antidote for cyanide poisoning (Filvo et al., 1993). Nitrites occupy a unique position in human toxicology. They ubiquitous in the environment and can be formed from nitrogenous compound by microorganisms present in the soil, water, saliva and gastrointestinal tract (Chow and Hong, 2002). Moreover nitrite $\left(\mathrm{No}_{2}{ }^{-}\right)$is a contaminant of 
some water supplies, extensive use of nitrogenous fertilizer, decomposition of plants and sewage wastes, followed by leaching of nitrates into ground water (Wolff and Wasserman, 1972). Nitrite is widely consumed from the diet by animals and humans. However the largest contribution to exposure results from the in vivo conversion of exogenously derived nitrate to nitrite. Because of its potential to cause methaemoglobin (MetHb) formation at excessive levels of intake, nitrite is regulated in feed and water as an undesirable substance. Forages and contaminated water have been shown to contain high levels of nitrate and represent the largest contributor to nitrite exposure for food-producing animals (Cockburn et al., 2013). A human health risk assessment was also carried out taking into account all direct and indirect sources of nitrite from the human diet, including carry-over of nitrite in animal-based products such as milk, eggs and meat products. The FAO/WHO, Joint Expert Committee on Food Additive (JECFA) established acceptable daily intakes (ADIs) of $0-0.07 \mathrm{mg} \mathrm{kg}^{-1}$ body weight for $\mathrm{NaNO}_{2}$ (expressed as nitrite ion) (WHO, 2003). In order to protect animal and human health, the European Union, directive, 2002/32/EC on undesirable substances in animal feed, restricts the maximum content of nitrite in complete feeding stuff (with a moisture content of $12 \%$ ) for livestock excluding birds and aquarium fish to $15 \mathrm{mg} / \mathrm{kg}$, and the maximum content of fish meal to $60 \mathrm{mg} / \mathrm{kg}$ (EC, 2002).

The hazardous effect of $\mathrm{NaNO}_{2}$ is derived from the reaction of nitrite with amines and amides to produce nitrosamines and nitrosamides, respectively. The toxic effects of nitrates and nitrites are well documented in mammalians, including impairment of reproductive function, hepatotoxicity and methaemoglobinemia, dysregulation of inflammatory responses and tissue injury, growth retardation and endocrine disturbance (Jahries et al., 1986). Nitrite is, however, also a natural constituent in the body, it is a potential nitric oxide (NO) donor and recent research has suggested that nitrite has important biological functions at low concentrations (Jensen, 2007). Nitric oxide free radical (NO) is generated from the nitrite by non-enzymatic method and $\mathrm{O}^{\circ}$ formation is found in acidic environments such as the stomach and oral cavity (Lundberg $\boldsymbol{e t}$ al., 1994 and McKnight $\boldsymbol{e t}$ al., 1997). Peroxy nitrite (ONOO'), the reaction product formed between $\mathrm{NO}^{\circ}$ and superoxide plays a critical role in the induction of inflammatory reaction and apoptosis. $\mathrm{O}^{\circ}$ is also associated with tumor promotion and/or progression (Radi et al., 1991). Anyway, nitrite when present at high concentration in blood, it can react with iron (III) of the hemoglobin, forming MetHb which has no oxygen-carrying ability. This fatal disease is called methemoglobinaemia (Zatar et al., 1999 and SanchezEchaniz et al., 2001). The use of permitted colors has raised concern that they might be used in excess of the saturatory limit $\left(100 \mathrm{mg} \mathrm{kg}^{-1}\right)$ or in foods in which they were not permitted (Rao and Bhat, 2003).

An interest in natural antioxidants, especially of vegetal origin, has greatly increased in recent years. Natural antioxidants can protect the human body from free radicals that may lead to the aging process and cause some chronic diseases including cancer, cardiovascular diseases and cataract as well as retard lipid oxidative rancidity in foods (Craig and Beck, 1999 and Lai et al., 2001).

Grape, one of the world's largest fruit crops, with more than 60 million tons is cultivated mainly as Vitis vinifera for wine production (Amico et al., 2004). It is estimated that around $13 \%$ of the total weight of grapes used for the wine and juice making results in grape pomace, which is a byproduct in this process (Torres et al., 2002). One way of using the potential of the grape pomace is the isolation of seeds and extraction of the polyphenols, since the total extractable phenolics in grape are a maximum 10\% in pulp, 60 to $70 \%$ in the seeds and 28 to $35 \%$ in the skin (Shi et al., 2003). Phenolic compounds from grape seeds, as other phenolic compounds have pharmacological and nutraceutical benefits both antiviral and antimutagenic (Saito et al., 1998). 
Grape seed extract (GSE) is a natural extract from the seeds of Vitis vinifera. A multitude of flavonoids are contained in GSE. The most abundant of these are the proanthocyanidins, which are oligomers of monomeric flavan-3-ol units linked by carboncarbon bonds. The major flavan-3-ols identified in GSE are (+)-catechin, (-)-epicatechin, and (-)-epicatechin-3-O-gallate (Santos-Buelga et al., 1995 and Waterhouse and Walzem, 1998).

It has proved that, free radical scavenging capacity of GSE is 20 times more effective than vitamin E and 50 times more effective than vitamin C. GSE is beneficial in many areas of health because of its antioxidant effect to bond with collagen, promoting youthful skin, cell health, elasticity, and flexibility. Moreover, proanthocyanidins help to protect the body from sun damage, to improve vision, to improve flexibility in joints, arteries, and body tissues such as the heart, and to improve blood circulation by strengthening capillaries, arteries, and veins. The most abundant phenolic compounds isolated from grape seed are catechins, epicatechin, procyanidin, and some dimers and trimers (Shi et al., 2003).

Here, we investigated the role of GSE in ameliorating $\mathrm{NaNO}_{2}$-induced abnormalities in the hematological and biochemical parameters and injury of body organs associated with oxidative stress in male albino rats.

\section{MATERIALS AND METHODS}

\section{Chemicals}

$\mathrm{NaNO}_{2}$ were obtained from Sigma Chemical Co. (St Louis, MO, USA) and all other chemicals and solvents were of analytical grade and purchased from Merck (Darmstadt, Germany), unless stated otherwise. Kits used for the estimation of GSH, GST, SOD and MDA were purchased from Biodiagnostic, France.

\section{Plant materials}

Dried grape seeds were bought from Harrase Company Giza, Egypt. The dried seeds were ground to a fine powder with electric blender, and kept at $4{ }^{\circ} \mathrm{C}$ until further use.

\section{Hydroalcoholic extract of dried grape seeds}

About $500 \mathrm{~g}$ of the powder was mixed with $1000 \mathrm{ml}$ of $70 \%$ ethanol in distilled water and kept for 3 days at room temperature. The extract was then filtered through a Buchner funnel. The filtrate was evaporated at $45{ }^{\circ} \mathrm{C}$ in a rotary evaporator to concentrate the solution, then lyophilized in order to obtain the dry extract and stored at $4{ }^{\circ} \mathrm{C}$ until use (Hemmati et al., 2008).

Enough amounts of the dried extract were suspended in water and administered orally by gavages to rats for 8 weeks $(150 \mathrm{mg} / \mathrm{kg} /$ day $)$.

\section{Animal and treatment}

Thirty-six male albino rats weighing about 130-140g (provided by the Laboratory Animal Center, Faculty of Veterinary Medicine, Cairo University) were housed in stainless steel cages in animal house in Regional Center for Food and Feed, Agricultural Research Center, Ministry of Agriculture, Giza, Egypt under controlled light and temperature conditions (12-h light/dark cycle, $22 \pm 2{ }^{\circ} \mathrm{C}$ ). During the acclimation period (1 week) and experimental period ( 8 weeks), the normal basal diet was supplied ad libitum. The basal diet consisted of casein $20 \%$, corn oil $10 \%$, cellulose (wooden fibers) $5 \%$, salt mixture $4 \%$, vitamin mixture $1 \%$ and starch $60 \%$ (Lane - Peter and Pearson, 1971). Rats were divided 
into six groups and treated for 8 week as follow: G1(normal control): Rats received purified tap water; G2: Rats received purified tap water + GSE $(150 \mathrm{mg} / \mathrm{kg}$ B.W/day, orally) (Hemmati et al., 2008); G3 (1g/L $\left.\mathrm{NaNO}_{2}\right)$ : Rats provided with purified drinking water containing $1 \mathrm{~g} / \mathrm{L} \mathrm{NaNO}_{2}$ (Mirvish et al., 1972); $\mathrm{G} 4\left(2 \mathrm{~g} / \mathrm{L} \mathrm{NaNO}_{2}\right)$ : Rats provided with purified drinking water containing $2 \mathrm{~g} / \mathrm{L} \mathrm{NaNO}_{2}$ (Subramaniyan et al., 2011); G5 $\left(\mathrm{GSE}+1 \mathrm{~g} / \mathrm{L} \mathrm{NaNO}_{2}\right)$ : Rats provided with purified drinking water containing $1 \mathrm{~g} / \mathrm{L} \mathrm{NaNO}_{2}+$ GSE (150 mg/kg B.W/day, orally) and G6 $\left(\mathrm{GSE}+2 \mathrm{~g} / \mathrm{L} \mathrm{NaNO}_{2}\right)$ : Rats provided with purified drinking water containing $2 \mathrm{~g} / \mathrm{L} \mathrm{NaNO}_{2}+\mathrm{GSE}(150 \mathrm{mg} / \mathrm{kg} \mathrm{B}$.W/day, orally).

At the $4^{\text {th }}$ week and $8^{\text {th }}$ week of the treatment period, blood samples were collected from the retro-orbital venous plexus under carbon dioxide anesthesia into three tubes. Two contain heparin, one used as fresh as possible for hematological parameters and other to obtain plasma. The third tube contains no anticoagulant to obtain serum. Plasma and serum separated and stored at $-20^{\circ} \mathrm{C}$ until analysis. Rats were scarified at the end of experiment and livers, kidneys, brains, hearts, lungs and testes were collected and kept for histological examination.

\section{Hematological analysis}

Whole blood was analyzed for: $\mathrm{Hb}, \mathrm{RBCs}$ and WBCs using an animal blood veterinary counter (ABC vet user Manual, RABOSIA Inc. A, France).

\section{Estimation of methemoglobin (MetHb)}

Aliquots of fresh blood were hemolyzed in water (1:5.5, v/v). After $3 \mathrm{~min}$, phosphate buffer was added, and the samples were centrifuged at $14,000 \mathrm{rpm}$ for $15 \mathrm{~min}$. Measurements of MetHb in hemolysates was based on the absorbance of MetHb spectrophotometrically at $630 \mathrm{~nm}$. Addition of cyanide eliminates the contribution of methemoglobin to the absorbance at $630 \mathrm{~nm}$. The absorbance of the hemolysate at $630 \mathrm{~nm}$ was first determined in the absence of cyanide. To $100 \mu 1$ of the hemolysate was added $50 \mu 1$ of a neutralized cyanide solution containing $5.3 \% \mathrm{w} / \mathrm{v}$ sodium cyanide and $5.6 \% \mathrm{v} / \mathrm{v}$ acetic acid, and the absorbance of the cyanide-treated sample was reread at $630 \mathrm{~nm}$. The absorbance in the absence of cyanide minus that in the presence of cyanide is a measure of the conversion of MetHb in the sample to cyanomethemoglobin which is proportional to concentration of MetHb (Kohn et al., 2002).

\section{Biochemical assays}

The ACE Alera Clinical Chemistry System Automatic Analyzer (Alfa Wasserman Corporation) was employed to measure the following parameters: ALT, AST, ALP, GGT, $\mathrm{TP}$, albumin, creatinine, urea, total cholesterol, triglycerides and glucose in serum.

\section{Estimation of antioxidant profile}

The extent of lipid peroxidation in terms of thiobarbituric acid reactive substances (TBARS) which react with malondialdehyde (MDA) in acidic medium was measured in serum calorimetrically at $534 \mathrm{~nm}$ according to the method of Onkawa et al. (1979). Plasma GSH was estimated calorimetrically at $405 \mathrm{~nm}$ based on the reduction of 5, 5 dithiobis (2nitrobenzoic acid) (DTNB) with GSH to produce yellow compound which directly proportional to GSH concentration (Beutler et al., 1963). Plasma GST was determined spectrophotometrically by measuring the conjugation of 1-chloro-2,4- dinitobenzene (CDNB) with reduced glutathione. The conjucation is accompined by an increase absorbance at $340 \mathrm{~nm}$. The rate of increase is directly proportional to the GST activity (Habig $\boldsymbol{e t}$ al., 1974). Plasma SOD activity was estimated spectrophotometrically at $560 \mathrm{~nm}$ based on the method described by Nishikimi et al. (1972) which relies on the ability of the enzyme to inhibite the phenazine methosulphate-mediated reduction of nitroblue tetrazolium dye. 


\section{Histopathological studies:}

Livers, kidneys, brains, hearts, lungs and testes from each group were fixed in $10 \%$ buffered formalin, routinely processed and embedded in paraffin. Sections of 4-6 $\mu \mathrm{m}$ thickness were prepared and stained with Haematoxylin and Eosin. Sections were then examined for histopathological findings (Banchroft et al., 1996).

\section{Statistical analysis:}

Statistical analysis of the obtained data was done using the least significant difference test (LSD) at the 5\% level of probability as outlined by Snedecor and Cochran (1980). Using the Duncan test institute program used a computer in the statistical analysis.

\section{RESULTS}

In this study, the data as shown in tables (1-7) recorded that, the administration of GSE alone (G2) did not induce any obvious changes in all examined parameters along experimental periods (comparing to control (G1) $(P<0.05)$ ).

\section{Hematological analysis:}

Results presented in table (1) demonstrated that $\mathrm{NaNO}_{2}$ administration for period of 4 and 8 weeks at both 2 dose levels (rats of G3 and G4) resulted in a significant dosedependent reduction $(P<0.05)$ of blood WBCs, RBCs count and $\mathrm{Hb}$ concentration. However, supplementation of $\mathrm{NaNO}_{2}$-intoxicated rats with GSE (G5 and G6) ameliorated the adverse effect of both $\mathrm{NaNO}_{2}$ doses on hematological parameters especially $1 \mathrm{~g} / \mathrm{L} \mathrm{NaNO}_{2}$ and at $8^{\text {th }}$ week of experiment. Whereas, GSE administration restore RBCs count to normal in blood of rats treated with $1 \mathrm{~g} / \mathrm{L} \mathrm{NaNO}_{2}$ at $8^{\text {th }}$ week of treatment (comparing with control $(P<0.05)$ ).

\section{Methemoglobin concentration:}

$\mathrm{MetHb}$ concentration was significantly elevated (corresponding to control group $(P<0.05))$ in time and dose dependant manner in blood of $\mathrm{NaNO}_{2}$-treated rats of $\mathrm{G} 3$ and $\mathrm{G} 4$ as shown in table (1). Meanwhile, co-administration of GSE with $1 \mathrm{~g}$ and $2 \mathrm{~g} / \mathrm{L}$ of $\mathrm{NaNO}_{2}$ (G5 and G6 respectively) resulted in attenuation of MetHb formation (compared to corresponding control $(P<0.05)$ ) in all experimental periods. Moreover, GSE was able to block MetHb formation in blood of $1 \mathrm{~g} \mathrm{NaNO}_{2}$ treated rats and restore it to normal level comparing with control group (G1).

\section{Liver function parameters}

Table (2) show the effect of GSE administration on liver function in serum of rats treated with $\mathrm{NaNO}_{2}$. Analysis of variance indicated that there was a significant dose dependant-increase in ALT, AST, ALP and GGT activities, whereas, a significant dose dependant-decrease in TP and albumin concentrations was detected in rats treated with both $\mathrm{NaNO}_{2}$ doses for 4 and 8 weeks. However, GSE administration in $\mathrm{NaNO}_{2}$-treated rats ameliorated the $\mathrm{NaNO}_{2}$ adverse effect as evidenced by a significant decrease of ALT, AST, ALP and GGT activities, and increase in TP and albumin concentration especially at low $\mathrm{NaNO}_{2}$ dose and at $8^{\text {th }}$ week. The activity of GGT in serum of rats co-administrated with GSE and $\mathrm{NaNO}_{2}$ was return to normal at $8^{\text {th }}$ week of treatment (compared with control $(P<0.05))$. 
Table (1): Hematological parameters and methemoglobin concentration in blood of control and treated rats during experimental periods (means $\pm \mathrm{SE}$ ).

\begin{tabular}{|c|c|c|c|c|c|c|c|c|}
\hline \multirow{2}{*}{ Groups } & \multicolumn{2}{|c|}{ WBCs $\left(10^{3} / \mu \mathrm{L}\right)$} & \multicolumn{2}{|c|}{ RBCs $\left(10^{6} / \mu \mathrm{L}\right)$} & \multicolumn{2}{|c|}{$\mathrm{Hb}(\mathrm{g} / \mathrm{dL})$} & \multicolumn{2}{|c|}{ MetHb (g\%) } \\
\hline & $\begin{array}{c}4 \\
\text { weeks }\end{array}$ & $\begin{array}{c}8 \\
\text { weeks }\end{array}$ & $\begin{array}{c}4 \\
\text { weeks }\end{array}$ & $\begin{array}{c}8 \\
\text { weeks }\end{array}$ & $\begin{array}{c}4 \\
\text { weeks }\end{array}$ & $\begin{array}{c}8 \\
\text { weeks }\end{array}$ & $\begin{array}{c}4 \\
\text { weeks }\end{array}$ & 8 weeks \\
\hline $\begin{array}{l}\text { G1 } \\
\text { Control }\end{array}$ & $\begin{array}{l}20.33 \\
\pm 0.33^{\mathrm{a}}\end{array}$ & $\begin{array}{l}21.33 \\
\pm 0.33^{\mathrm{a}}\end{array}$ & $\begin{array}{c}11.33 \\
\pm 0.33^{\mathrm{a}}\end{array}$ & $\begin{array}{l}11.67 \\
\pm 0.88^{\mathrm{a}}\end{array}$ & $\begin{array}{c}16.51 \\
\pm 0.29^{\mathrm{a}}\end{array}$ & $\begin{array}{c}17.36 \\
\pm 0.19^{\mathrm{a}}\end{array}$ & $\begin{array}{c}0.34 \\
\pm 0.01^{\mathrm{e}}\end{array}$ & $\begin{array}{c}0.34 \\
\pm 0.01^{\mathrm{de}}\end{array}$ \\
\hline $\begin{array}{l}\text { G2 } \\
\text { GSE }\end{array}$ & $\begin{array}{l}21.00 \\
\pm 0.58^{\mathrm{a}}\end{array}$ & $\begin{array}{l}21.67 \\
\pm 0.67^{\mathrm{a}}\end{array}$ & $\begin{array}{r}10.97 \\
\pm 0.63^{\mathrm{a}}\end{array}$ & $\begin{array}{c}11.64 \\
\pm 0.20^{\mathrm{a}}\end{array}$ & $\begin{array}{c}16.35 \\
\pm 0.26^{\mathrm{a}}\end{array}$ & $\begin{array}{c}17.25 \\
\pm 0.15^{\mathrm{a}}\end{array}$ & $\begin{array}{c}0.34 \\
\pm 0.01^{\mathrm{e}}\end{array}$ & $\begin{array}{c}0.33 \\
\pm 0.01^{\mathrm{e}}\end{array}$ \\
\hline $\begin{array}{l}\mathrm{G} 3 \\
1 \mathrm{~g} / \mathrm{L} \\
\mathrm{NaNO}_{2}\end{array}$ & $\begin{array}{r}16.33 \\
\pm 0.33^{\mathrm{c}}\end{array}$ & $\begin{array}{c}16.33 \\
\pm 0.33^{\mathrm{c}}\end{array}$ & $\begin{array}{c}8.39 \\
\pm 0.16^{\mathrm{c}}\end{array}$ & $\begin{array}{r}7.87 \\
\pm 0.18^{\mathrm{c}}\end{array}$ & $\begin{array}{c}14.37 \\
\pm 0.27^{\mathrm{c}}\end{array}$ & $\begin{array}{c}13.29 \\
\pm 0.14^{\mathrm{d}}\end{array}$ & $\begin{array}{c}0.96 \\
\pm 0.01^{\mathrm{c}}\end{array}$ & $\begin{array}{c}1.62 \\
\pm 0.02^{\mathrm{b}}\end{array}$ \\
\hline $\begin{array}{l}\mathrm{G} 4 \\
2 \mathrm{~g} / \mathrm{L} \\
\mathrm{NaNO}_{2}\end{array}$ & $\begin{array}{c}14.33 \\
\pm 0.33^{\mathrm{d}}\end{array}$ & $\begin{array}{c}13.00 \\
\pm 0.58^{\mathrm{d}}\end{array}$ & $\begin{array}{c}6.88 \\
\pm 0.20^{\mathrm{d}}\end{array}$ & $\begin{array}{c}6.00 \\
\pm 0.07^{\mathrm{d}}\end{array}$ & $\begin{array}{c}13.23 \\
\pm 0.15^{\mathrm{d}}\end{array}$ & $\begin{array}{c}11.14 \\
\pm 0.25^{\mathrm{e}}\end{array}$ & $\begin{array}{c}1.52 \\
\pm 0.01^{\mathrm{a}}\end{array}$ & $\begin{array}{c}2.43 \\
\pm 0.04^{\mathrm{a}}\end{array}$ \\
\hline $\begin{array}{l}\mathrm{G} 5 \\
\mathrm{GSE}+1 \mathrm{~g} / \mathrm{L} \\
\mathrm{NaNO}_{2}\end{array}$ & $\begin{array}{c}18.67 \\
\pm 0.33^{\mathrm{b}}\end{array}$ & $\begin{array}{c}19.67 \\
\pm 0.33^{\mathrm{b}}\end{array}$ & $\begin{array}{c}9.59 \\
\pm 0.10^{\mathrm{b}}\end{array}$ & $\begin{array}{r}10.90 \\
\pm 0.40^{\mathrm{a}}\end{array}$ & $\begin{array}{c}15.24 \\
\pm 0.12^{\mathrm{b}}\end{array}$ & $\begin{array}{c}16.61 \\
\pm 0.12^{\mathrm{b}}\end{array}$ & $\begin{array}{c}0.78 \\
\pm 0.02^{\mathrm{d}}\end{array}$ & $\begin{array}{c}0.39 \\
\pm 0.01^{\mathrm{d}}\end{array}$ \\
\hline $\begin{array}{l}\text { G6 } \\
\text { GSE+ 2g/L } \\
\mathrm{NaNO}_{2}\end{array}$ & $\begin{array}{r}16.67 \\
\pm 0.33^{\mathrm{c}}\end{array}$ & $\begin{array}{l}17.67 \\
\pm 0.33^{\mathrm{c}}\end{array}$ & $\begin{array}{c}8.36 \\
\pm 0.09^{\mathrm{c}}\end{array}$ & $\begin{array}{c}9.26 \\
\pm 0.13^{\mathrm{b}}\end{array}$ & $\begin{array}{c}14.08 \\
\pm 0.08^{\mathrm{c}}\end{array}$ & $\begin{array}{c}15.67 \\
\pm 0.19^{\mathrm{c}}\end{array}$ & $\begin{array}{c}1.19 \\
\pm 0.01^{\mathrm{b}}\end{array}$ & $\begin{array}{c}0.99 \\
\pm 0.01^{\mathrm{c}}\end{array}$ \\
\hline $\mathrm{LSD}_{0.05}$ & 1.186 & 1.390 & 0.716 & 1.048 & 0.646 & 0.549 & 0.039 & 0.054 \\
\hline
\end{tabular}

Within the same column, various superscript letters indicate significant differences (Duncan, $P<0.05$ ).

Kidney function parameters:

The kidney function parameters results summarized in table (3) revealed that there is a significant dose-dependent increase $(P<0.05)$ in creatinine and urea concentrations in serum of $\mathrm{NaNO}_{2}$-treated rats (G3 and G4) while GSE intake caused desperation of this increase, in addition GSE administration restore creatinine in serum of $1 \mathrm{~g} \mathrm{NaNO}_{2}$-treated rats (G5) to normal and restore urea level in serum of both groups of $\mathrm{NaNO}_{2}$-treated rats (G5 and G6) to normal at $8^{\text {th }}$ week of treatment (compared to corresponding control $(P<0.05)$ ).

\section{Lipid profile parameters:}

The data in table (4) shows that $\mathrm{NaNO}_{2}$ induced significant dose-dependent increase in serum total cholesterol and triglycerides concentrations comparing with control $(P<0.05)$ at all experimental periods. Simultaneous administrations of GSE and $\mathrm{NaNO}_{2}$ in G5 and G6 rats resulted in significant reduction of increased total cholesterol and triglycerides concentrations and restore cholesterol level at $4^{\text {th }}$ week of treatment and triglycerides levels along experimental period to normal in serum of $1 \mathrm{~g} \mathrm{NaNO}_{2}$-treated rats comparing with control $(P<0.05)$. 
Table (2): Liver functions parameters in serum of control and treated rats during experimental periods (means \pm SE).

\begin{tabular}{|c|c|c|c|c|c|c|c|c|c|c|c|c|}
\hline \multirow{2}{*}{$\begin{array}{l}\text { Parapeters } \\
\text { Groups }\end{array}$} & \multicolumn{2}{|c|}{ ALT (U/L) } & \multicolumn{2}{|c|}{ AST (U/L) } & \multicolumn{2}{|c|}{ ALP (U/L) } & \multicolumn{2}{|c|}{ GGT (U/L) } & \multicolumn{2}{|c|}{$\mathrm{TP}(\mathrm{g} / \mathrm{dL})$} & \multicolumn{2}{|c|}{ Albumin $(\mathrm{g} / \mathrm{dL})$} \\
\hline & 4 weeks & 8 weeks & 4 weeks & 8 weeks & 4 weeks & 8 weeks & 4 weeks & 8 weeks & 4 weeks & 8 weeks & 4 weeks & 8 weeks \\
\hline $\begin{array}{l}\text { G1 } \\
\text { Control }\end{array}$ & $\begin{array}{c}40.00 \\
\pm 0.58^{d}\end{array}$ & $\begin{array}{l}42.00 \\
\pm 0.58^{\mathrm{e}}\end{array}$ & $\begin{array}{l}93.33 \\
\pm 0.88^{\mathrm{e}}\end{array}$ & $\begin{array}{l}95.00 \\
\pm 0.24^{\mathrm{e}}\end{array}$ & $\begin{array}{r}359.00 \\
\pm 14.57^{\mathrm{e}}\end{array}$ & $\begin{array}{l}412.00 \\
\pm 4.36^{\mathrm{e}}\end{array}$ & $\begin{array}{c}2.33 \\
\pm 0.33^{\mathrm{d}}\end{array}$ & $\begin{array}{c}2.67 \\
\pm 0.33^{\mathrm{d}}\end{array}$ & $\begin{array}{c}6.76 \\
\pm 0.07^{\mathrm{a}}\end{array}$ & $\begin{array}{c}6.86 \\
\pm 0.04^{\mathrm{a}}\end{array}$ & $\begin{array}{c}4.14 \\
\pm 0.06^{\mathrm{a}}\end{array}$ & $\begin{array}{c}4.19 \\
\pm 0.02^{\mathrm{a}}\end{array}$ \\
\hline $\begin{array}{l}\text { G2 } \\
\text { GSE }\end{array}$ & $\begin{array}{l}41.00 \\
\pm 1.53^{\mathrm{d}}\end{array}$ & $\begin{array}{l}41.67 \\
\pm 1.20^{\mathrm{e}}\end{array}$ & $\begin{array}{r}92.90 \\
\pm 0.61^{\mathrm{e}}\end{array}$ & $\begin{array}{l}94.55 \\
\pm 0.55^{\mathrm{e}}\end{array}$ & $\begin{array}{r}367.00 \\
\pm 14.73^{\mathrm{e}}\end{array}$ & $\begin{array}{l}411.00 \\
\pm 6.66^{\mathrm{e}}\end{array}$ & $\begin{array}{c}2.33 \\
\pm 0.33^{\mathrm{d}}\end{array}$ & $\begin{array}{c}2.67 \\
\pm 0.33^{\mathrm{d}}\end{array}$ & $\begin{array}{c}6.74 \\
\pm 0.05^{\mathrm{a}}\end{array}$ & $\begin{array}{c}6.88 \\
\pm 0.06^{\mathrm{a}}\end{array}$ & $\begin{array}{c}4.11 \\
\pm 0.05^{\mathrm{a}}\end{array}$ & $\begin{array}{c}4.22 \\
\pm 0.02^{\mathrm{a}}\end{array}$ \\
\hline $\begin{array}{l}\mathrm{G} 3 \\
1 \mathrm{~g} / \mathrm{L} \\
\mathrm{NaNO}_{2} \\
\end{array}$ & $\begin{array}{c}55.33 \\
\pm 0.88^{b}\end{array}$ & $\begin{array}{l}62.67 \\
\pm 0.88^{\mathrm{b}}\end{array}$ & $\begin{array}{l}109.00 \\
\pm 0.31^{\mathrm{c}}\end{array}$ & $\begin{array}{l}128.00 \\
\pm 0.58^{\mathrm{b}}\end{array}$ & $\begin{array}{l}590.33 \\
\pm 3.84^{\mathrm{b}}\end{array}$ & $\begin{array}{l}627.67 \\
\pm 9.06^{b}\end{array}$ & $\begin{array}{c}5.33 \\
\pm 0.33^{\mathrm{b}}\end{array}$ & $\begin{array}{c}6.33 \\
\pm 0.33^{\mathrm{b}}\end{array}$ & $\begin{array}{c}5.69 \\
\pm 0.14^{\mathrm{c}}\end{array}$ & $\begin{array}{c}4.93 \\
\pm 0.08^{\mathrm{d}}\end{array}$ & $\begin{array}{c}3.32 \\
\pm 0.10^{\mathrm{c}}\end{array}$ & $\begin{array}{c}3.07 \\
\pm 0.03^{\mathrm{d}}\end{array}$ \\
\hline $\begin{array}{l}\mathrm{G} 4 \\
2 \mathrm{~g} / \mathrm{L} \\
\mathrm{NaNO}_{2}\end{array}$ & $\begin{array}{c}67.00 \\
\pm 1.53^{\mathrm{a}}\end{array}$ & $\begin{array}{l}81.00 \\
\pm 1.15^{\mathrm{a}}\end{array}$ & $\begin{array}{l}121.67 \\
\pm 1.20^{\mathrm{a}}\end{array}$ & $\begin{array}{l}140.67 \\
\pm 1.20^{\mathrm{a}}\end{array}$ & $\begin{array}{l}623.33 \\
\pm 5.70^{\mathrm{a}}\end{array}$ & $\begin{array}{l}706.33 \\
\pm 3.84^{\mathrm{a}}\end{array}$ & $\begin{array}{c}6.67 \\
\pm 0.33^{\mathrm{a}}\end{array}$ & $\begin{array}{c}8.33 \\
\pm 0.33^{\mathrm{a}}\end{array}$ & $\begin{array}{c}4.71 \\
\pm 0.10^{\mathrm{d}}\end{array}$ & $\begin{array}{c}4.07 \\
\pm 0.09^{\mathrm{e}}\end{array}$ & $\begin{array}{c}3.10 \\
\pm 0.03^{\mathrm{d}}\end{array}$ & $\begin{array}{c}2.84 \\
\pm 0.03^{\mathrm{e}}\end{array}$ \\
\hline $\begin{array}{l}\mathrm{G} 5 \\
\mathrm{GSE}+1 \mathrm{~g} / \mathrm{L} \\
\mathrm{NaNO}_{2}\end{array}$ & $\begin{array}{c}50.00 \\
\pm 0.58^{c}\end{array}$ & $\begin{array}{l}45.00 \\
\pm 0.58^{\mathrm{d}}\end{array}$ & $\begin{array}{l}105.00 \\
\pm 0.57^{\mathrm{d}}\end{array}$ & $\begin{array}{c}99.19 \\
\pm 0.33^{\mathrm{d}}\end{array}$ & $\begin{array}{l}489.67 \\
\pm 2.03^{d}\end{array}$ & $\begin{array}{l}436.33 \\
\pm 3.28^{d}\end{array}$ & $\begin{array}{c}3.67 \\
\pm 0.33^{\mathrm{c}}\end{array}$ & $\begin{array}{c}3.33 \\
\pm 0.33^{\mathrm{d}}\end{array}$ & $\begin{array}{c}6.06 \\
\pm 0.07^{b}\end{array}$ & $\begin{array}{c}6.45 \\
\pm 0.04^{\mathrm{b}}\end{array}$ & $\begin{array}{c}3.71 \\
\pm 0.03^{\mathrm{b}}\end{array}$ & $\begin{array}{c}4.04 \\
\pm 0.06^{\mathrm{b}}\end{array}$ \\
\hline $\begin{array}{l}\mathrm{G} 6 \\
\mathrm{GSE}+2 \mathrm{~g} / \mathrm{L} \\
\mathrm{NaNO}_{2}\end{array}$ & $\begin{array}{c}57.67 \\
\pm 1.45^{\mathrm{b}}\end{array}$ & $\begin{array}{l}51.00 \\
\pm 0.58^{\mathrm{c}}\end{array}$ & $\begin{array}{l}112.66 \\
\pm 0.67^{b}\end{array}$ & $\begin{array}{l}105.33 \\
\pm 0.34^{\mathrm{c}}\end{array}$ & $\begin{array}{c}555.33 \\
\pm 12.20^{c}\end{array}$ & $\begin{array}{l}510.67 \\
\pm 7.88^{\mathrm{c}}\end{array}$ & $\begin{array}{c}5.67 \\
\pm 0.33^{\mathrm{b}}\end{array}$ & $\begin{array}{c}4.67 \\
\pm 0.33^{\mathrm{c}}\end{array}$ & $\begin{array}{c}5.42 \\
\pm 0.08^{c}\end{array}$ & $\begin{array}{c}6.02 \\
\pm 0.13^{\mathrm{c}}\end{array}$ & $\begin{array}{c}3.48 \\
\pm 0.01^{\mathrm{c}}\end{array}$ & $\begin{array}{c}3.93 \\
\pm 0.04^{\mathrm{c}}\end{array}$ \\
\hline $\mathrm{LSD}_{0.05}$ & 3.607 & 2.685 & 2.340 & 1.856 & 31.560 & 19.206 & 0.938 & 1.027 & 0.279 & 0.239 & 0.168 & 0.108 \\
\hline
\end{tabular}

Within the same column, various superscript letters indicate significant differences (Duncan, $P<0.05$ ). 
Table (3): Kidney functions parameters in serum of control and treated rats during experimental periods (means $\pm \mathrm{SE}$ ).

\begin{tabular}{|l|c|c|c|c|}
\hline \multirow{2}{*}{ Parameters } & \multicolumn{2}{|c|}{ Creatinine $(\mathrm{mg} / \mathrm{dL})$} & \multicolumn{2}{c|}{ Urea $(\mathrm{mg} / \mathrm{dL})$} \\
\cline { 2 - 5 } & 4 weeks & 8 weeks & 4 weeks & 8 weeks \\
\hline $\begin{array}{l}\mathrm{G} 1 \\
\text { Control }\end{array}$ & $0.57 \pm 0.03^{\mathrm{c}}$ & $0.58 \pm 0.01^{\mathrm{b}}$ & $36.33 \pm 0.88^{\mathrm{d}}$ & $37.67 \pm 0.88^{\mathrm{c}}$ \\
\hline $\begin{array}{l}\mathrm{G} 2 \\
\mathrm{GSE}\end{array}$ & $0.58 \pm 0.02^{\mathrm{c}}$ & $0.58 \pm 0.01^{\mathrm{b}}$ & $36.00 \pm 1.15^{\mathrm{d}}$ & $37.00 \pm 1.53^{\mathrm{c}}$ \\
\hline $\begin{array}{l}\mathrm{G} 3 \\
1 \mathrm{~g} / \mathrm{L} \mathrm{NaNO} \mathrm{Na}_{2}\end{array}$ & $0.73 \pm 0.03^{\mathrm{b}}$ & $0.80 \pm 0.06^{\mathrm{a}}$ & $45.00 \pm 0.58^{\mathrm{b}}$ & $59.00 \pm 1.15^{\mathrm{b}}$ \\
\hline $\begin{array}{l}\mathrm{G} 4 \\
2 \mathrm{~g} / \mathrm{L} \mathrm{NaNO} \mathrm{NaN}_{2}\end{array}$ & $0.90 \pm 0.06^{\mathrm{a}}$ & $0.93 \pm 0.09^{\mathrm{a}}$ & $55.33 \pm 1.76^{\mathrm{a}}$ & $69.00 \pm 0.58^{\mathrm{a}}$ \\
\hline $\begin{array}{l}\mathrm{G} 5 \\
\mathrm{GSE}+1 \mathrm{~g} / \mathrm{L} \\
\mathrm{NaNO}_{2}\end{array}$ & $0.73 \pm 0.03^{\mathrm{b}}$ & $0.63 \pm 0.02^{\mathrm{b}}$ & $40.00 \pm 0.58^{\mathrm{c}}$ & $38.00 \pm 0.58^{\mathrm{c}}$ \\
\hline $\begin{array}{l}\mathrm{G} 6 \\
\mathrm{GSE}+2 \mathrm{~g} / \mathrm{L} \\
\mathrm{NaNO}_{2}\end{array}$ & $0.87 \pm 0.02^{\mathrm{a}}$ & $0.80 \pm 0.05^{\mathrm{a}}$ & $42.67 \pm 1.20^{\mathrm{c}}$ & $40.00 \pm 1.15^{\mathrm{c}}$ \\
\hline $\mathrm{LSD}_{0.05}$ & 0.107 & 0.149 & 3.406 & 3.193 \\
\hline
\end{tabular}

Within the same column, various superscript letters indicate significant differences (Duncan, $P<0.05$ ).

Table (4): Total cholesterol and triglycerides concentrations in serum of control and treated rats during experimental periods (means $\pm \mathrm{SE}$ ).

\begin{tabular}{|l|c|c|c|c|}
\hline \multirow{2}{*}{\begin{tabular}{l} 
Garameters \\
\cline { 2 - 5 }
\end{tabular}} & \multicolumn{2}{|c|}{ Cholesterol $(\mathrm{mg} / \mathrm{dL})$} & \multicolumn{2}{c|}{ Triglycerides $(\mathrm{mg} / \mathrm{dL})$} \\
\cline { 2 - 5 } $\begin{array}{l}\mathrm{G} 1 \\
\text { Control }\end{array}$ & $37.67 \pm 1.20^{\mathrm{d}}$ & $38.33 \pm 1.20^{\mathrm{d}}$ & $106.00 \pm 1.15^{\mathrm{c}}$ & $107.00 \pm 1.15^{\mathrm{d}}$ \\
\hline $\begin{array}{l}\mathrm{G} 2 \\
\mathrm{GSE}\end{array}$ & $37.00 \pm 1.15^{\mathrm{d}}$ & $37.67 \pm 0.88^{\mathrm{d}}$ & $105.00 \pm 1.73^{\mathrm{c}}$ & $105.33 \pm 1.76^{\mathrm{d}}$ \\
\hline $\begin{array}{l}\mathrm{G} 3 \\
1 \mathrm{~g} / \mathrm{L} \mathrm{NaNO} \mathrm{NaN}_{2}\end{array}$ & $50.33 \pm 0.88^{\mathrm{b}}$ & $70.33 \pm 0.88^{\mathrm{b}}$ & $127.00 \pm 1.73^{\mathrm{b}}$ & $138.33 \pm 1.45^{\mathrm{b}}$ \\
\hline $\begin{array}{l}\mathrm{G} 4 \\
2 \mathrm{~g} / \mathrm{L} \mathrm{NaNO} \mathrm{NaN}_{2}\end{array}$ & $61.67 \pm 1.45^{\mathrm{a}}$ & $90.00 \pm 1.15^{\mathrm{a}}$ & $136.00 \pm 1.53^{\mathrm{a}}$ & $146.67 \pm 1.86^{\mathrm{a}}$ \\
\hline $\begin{array}{l}\mathrm{G} 5 \\
\mathrm{GSE}+1 \mathrm{~g} / \mathrm{L} \\
\mathrm{NaNO}_{2}\end{array}$ & $45.33 \pm 1.20^{\mathrm{c}}$ & $40.00 \pm 0.58^{\mathrm{d}}$ & $108.67 \pm 1.20^{\mathrm{c}}$ & $107.67 \pm 0.88^{\mathrm{d}}$ \\
\hline $\begin{array}{l}\mathrm{G} 6 \\
\mathrm{GSE}+2 \mathrm{~g} / \mathrm{L} \\
\mathrm{NaNO}_{2}\end{array}$ & $52.00 \pm 1.15^{\mathrm{b}}$ & $47.33 \pm 0.88^{\mathrm{c}}$ & $124.00 \pm 2.31^{\mathrm{b}}$ & $122.33 \pm 1.20^{\mathrm{c}}$ \\
\hline $\mathrm{LSD}_{0.05}$ & 3.655 & 2.935 & 5.10 & 4.397 \\
\hline
\end{tabular}

Within the same column, various superscript letters indicate significant differences (Duncan, $P<0.05$ ). 


\section{Glucose concentration}

As shown in table (5) $\mathrm{NaNO}_{2}$ treatment induced significant dose-dependent increase in serum glucose level in respect to control group $(P<0.05)$. The administration of GSE significantly alleviated the hyperglycemic effect of $\mathrm{NaNO}_{2}$. Such effect was more pronounced with $1 \mathrm{~g} \mathrm{NaNO}_{2}$ and seemed to be time dependent as glucose level was return to normal in rats co-administrated with $1 \mathrm{~g} \mathrm{NaNO}_{2}$ and GSE at $8^{\text {th }}$ week of treatment $(P<0.05)$.

Table (5): Glucose concentrations in serum of control and treated rats during experimental periods (means $\pm \mathrm{SE}$ ).

\begin{tabular}{|l|c|c|}
\multirow{2}{*}{ Groups } & \multicolumn{2}{|c|}{ Glucose $(\mathrm{mg} / \mathrm{dL})$} \\
\cline { 2 - 3 } & 4 weeks & 8 weeks \\
\hline $\begin{array}{l}\text { G1 } \\
\text { Control }\end{array}$ & $109.33 \pm 2.19^{\mathrm{e}}$ & $113.33 \pm 2.19^{\mathrm{de}}$ \\
$\mathrm{GSE}$ & $108.67 \pm 4.63^{\mathrm{e}}$ & $106.00 \pm 4.62^{\mathrm{e}}$ \\
\hline $\begin{array}{l}\mathrm{G} 3 \\
1 \mathrm{~g} / \mathrm{L} \mathrm{NaNO}\end{array}$ & $138.00 \pm 1.15^{\mathrm{c}}$ & $144.67 \pm 1.20^{\mathrm{b}}$ \\
\hline $\begin{array}{l}\mathrm{G} 4 \\
2 \mathrm{~g} / \mathrm{L} \mathrm{NaNO}\end{array}$ & $170.67 \pm 1.20^{\mathrm{a}}$ & $181.00 \pm 1.53^{\mathrm{a}}$ \\
\hline $\begin{array}{l}\mathrm{G} 5 \\
\mathrm{GSE}+1 \mathrm{~g} / \mathrm{L} \mathrm{NaNO}_{2}\end{array}$ & $121.33 \pm 1.45^{\mathrm{d}}$ & $114.33 \pm 2.19^{\mathrm{d}}$ \\
\hline $\begin{array}{l}\mathrm{G} 6 \\
\mathrm{GSE}+2 \mathrm{~g} / \mathrm{L} \mathrm{NaNO}_{2}\end{array}$ & $148.33 \pm 1.20^{\mathrm{b}}$ & $122.67 \pm 1.86^{\mathrm{c}}$ \\
\hline $\mathrm{LSD} 0.05$ & 7.177 & 7.766 \\
\hline
\end{tabular}

Within the same column, various superscript letters indicate significant differences (Duncan, $P<0.05$ ).

\section{Antioxidant profile:}

Data in table (6) illustrated that $\mathrm{NaNO}_{2}$ treatment significantly reduce $(P<0.05)$ plasma GSH, GST and SOD levels in dose-dependent manner in all experimental periods. GSE administration significantly elevate of GSH, GST and SOD levels in plasma of $\mathrm{NaNO}_{2-}$ intoxicated rats especially with $1 \mathrm{~g} \mathrm{NaNO}_{2}$ dose (G5) and at $8^{\text {th }}$ week of experiment comparing with corresponding control $(P<0.05)$. GSH and GST levels were restored to normal level at $8^{\text {th }}$ week of experiment as a result of GSE intake in $1 \mathrm{~g} \mathrm{NaNO}$-treated rats.

Serum MDA level (lipid peroxidation product) was increased parallel to dose and time of $\mathrm{NaNO}_{2}$ intake in $\mathrm{G} 3$ and $\mathrm{G} 4$ rats comparing with control $(P<0.05)$. The administration of GSE ameliorated the $\mathrm{NaNO}_{2}$-induced elevation in lipid peroxidation as evidenced by a significant decrease $(P<0.05)$ in MDA production in G5 and G6 rats. Such effect was more pronounced with $1 \mathrm{~g} \mathrm{NaNO}_{2}$ and seemed to be time dependent as compared to control rats. The results were shown in table (7). 
Table (6): Antioxidants parameters in plasma of control and treated rats during experimental periods (means $\pm \mathrm{SE}$ ).

\begin{tabular}{|c|c|c|c|c|c|c|}
\hline \multirow{2}{*}{ Groups } & \multicolumn{2}{|c|}{ GSH (mg/dL) } & \multicolumn{2}{|c|}{ GST (u/ml) } & \multicolumn{2}{|c|}{$\mathrm{SOD}(\mathrm{u} / \mathrm{ml})$} \\
\hline & 4 weeks & 8 weeks & 4 weeks & 8 weeks & 4 weeks & 8 weeks \\
\hline $\begin{array}{l}\text { G1 } \\
\text { Control }\end{array}$ & $\begin{array}{c}0.53 \\
\pm 0.01^{\mathrm{a}}\end{array}$ & $\begin{array}{c}0.52 \\
\pm 0.01^{\mathrm{a}}\end{array}$ & $\begin{array}{l}61.33 \\
\pm 0.88^{\mathrm{a}}\end{array}$ & $\begin{array}{c}64.00 \\
\pm 0.58^{\mathrm{a}}\end{array}$ & $\begin{array}{l}542.23 \\
\pm 7.08^{\mathrm{a}}\end{array}$ & $\begin{array}{l}544.86 \\
\pm 8.67^{\mathrm{a}}\end{array}$ \\
\hline $\begin{array}{l}\text { G2 } \\
\text { GSE }\end{array}$ & $\begin{array}{c}0.54 \\
\pm 0.01^{\mathrm{a}}\end{array}$ & $\begin{array}{c}0.53 \\
\pm 0.02^{\mathrm{a}}\end{array}$ & $\begin{array}{l}61.32 \\
\pm 0.34^{\mathrm{a}}\end{array}$ & $\begin{array}{c}63.69 \\
\pm 0.35^{\mathrm{a}}\end{array}$ & $\begin{array}{l}528.99 \\
\pm 8.77^{\mathrm{a}}\end{array}$ & $\begin{array}{l}550.78 \\
\pm 9.66^{\mathrm{a}}\end{array}$ \\
\hline $\begin{array}{l}\mathrm{G} 3 \\
1 \mathrm{~g} / \mathrm{L} \mathrm{NaNO}_{2}\end{array}$ & $\begin{array}{c}0.42 \\
\pm 0.01^{\mathrm{c}}\end{array}$ & $\begin{array}{c}0.34 \\
\pm 0.01^{\mathrm{c}}\end{array}$ & $\begin{array}{c}58.29 \\
\pm 0.36^{\mathrm{b}}\end{array}$ & $\begin{array}{l}52.34 \\
\pm 0.18^{\mathrm{c}}\end{array}$ & $\begin{array}{l}436.86 \\
\pm 7.48^{\mathrm{c}}\end{array}$ & $\begin{array}{l}392.44 \\
\pm 5.23^{\mathrm{d}}\end{array}$ \\
\hline $\begin{array}{l}\mathrm{G} 4 \\
2 \mathrm{~g} / \mathrm{L} \mathrm{NaNO}_{2}\end{array}$ & $\begin{array}{c}0.34 \\
\pm 0.01^{\mathrm{d}}\end{array}$ & $\begin{array}{c}0.28 \\
\pm 0.01^{\mathrm{d}}\end{array}$ & $\begin{array}{l}55.00 \\
\pm 0.57^{\mathrm{c}}\end{array}$ & $\begin{array}{l}48.29 \\
\pm 0.30^{\mathrm{d}}\end{array}$ & $\begin{array}{l}336.55 \\
\pm 10.99^{d}\end{array}$ & $\begin{array}{l}263.44 \\
\pm 10.99^{\mathrm{e}}\end{array}$ \\
\hline $\begin{array}{l}\mathrm{G} 5 \\
\mathrm{GSE}+1 \mathrm{~g} / \mathrm{L} \\
\mathrm{NaNO}_{2}\end{array}$ & $\begin{array}{c}0.46 \\
\pm 0.01^{\mathrm{b}}\end{array}$ & $\begin{array}{c}0.50 \\
\pm 0.01^{\mathrm{a}}\end{array}$ & $\begin{array}{l}59.00 \\
\pm 0.58^{\mathrm{b}}\end{array}$ & $\begin{array}{l}63.11 \\
\pm 0.12^{\mathrm{a}}\end{array}$ & $\begin{array}{l}481.96 \\
\pm 2.23^{\mathrm{b}}\end{array}$ & $\begin{array}{l}503.04 \\
\pm 5.26^{\mathrm{b}}\end{array}$ \\
\hline $\begin{array}{l}\mathrm{G} 6 \\
\mathrm{GSE}+2 \mathrm{~g} / \mathrm{L} \\
\mathrm{NaNO}_{2} \\
\end{array}$ & $\begin{array}{c}0.40 \\
\pm 0.01^{\mathrm{c}}\end{array}$ & $\begin{array}{c}0.45 \\
\pm 0.01^{\mathrm{b}}\end{array}$ & $\begin{array}{l}57.65 \\
\pm 0.29^{b}\end{array}$ & $\begin{array}{c}60.27 \\
\pm 0.37^{b}\end{array}$ & $\begin{array}{l}426.69 \\
\pm 2.88^{c}\end{array}$ & $\begin{array}{l}471.35 \\
\pm 8.81^{\mathrm{c}}\end{array}$ \\
\hline $\mathrm{LSD}_{0.05}$ & 0.030 & 0.032 & 1.670 & 1.075 & 22.402 & 25.839 \\
\hline
\end{tabular}

Within the same column, various superscript letters indicate significant differences (Duncan, $P<0.05$ ).

Table (7): Lipid peroxidation (Malondialdehyde levels) in serum of control and treated rats during experimental periods (means $\pm \mathrm{SE}$ ).

\begin{tabular}{|c|c|c|}
\hline \multirow{2}{*}{ Groups } & \multicolumn{2}{|c|}{$\mathrm{MDA} \mathrm{nmol} / \mathrm{ml}$} \\
\hline & 4 weeks & 8 weeks \\
\hline $\begin{array}{l}\text { G1 } \\
\text { Control }\end{array}$ & $1.86 \pm 0.02^{\mathrm{e}}$ & $1.82 \pm 0.07^{\mathrm{e}}$ \\
\hline $\begin{array}{l}\text { G2 } \\
\text { GSE }\end{array}$ & $1.86 \pm 0.04^{\mathrm{e}}$ & $1.78 \pm 0.04^{\mathrm{e}}$ \\
\hline $\begin{array}{l}\mathrm{G} 3 \\
1 \mathrm{~g} / \mathrm{L} \mathrm{NaNO}_{2}\end{array}$ & $6.35 \pm 0.10^{\mathrm{b}}$ & $7.32 \pm 0.14^{\mathrm{b}}$ \\
\hline $\begin{array}{l}\mathrm{G} 4 \\
2 \mathrm{~g} / \mathrm{L} \mathrm{NaNO}_{2}\end{array}$ & $8.02 \pm 0.06^{\mathrm{a}}$ & $10.17 \pm 0.12^{a}$ \\
\hline $\begin{array}{l}\mathrm{G} 5 \\
\mathrm{GSE}+1 \mathrm{~g} / \mathrm{L} \mathrm{NaNO}_{2}\end{array}$ & $3.85 \pm 0.44^{\mathrm{d}}$ & $2.78 \pm 0.10^{\mathrm{d}}$ \\
\hline $\begin{array}{l}\mathrm{G} 6 \\
\mathrm{GSE}+2 \mathrm{~g} / \mathrm{L} \mathrm{NaNO} \\
\end{array}$ & $5.03 \pm 0.13^{c}$ & $3.65 \pm 0.13^{\mathrm{c}}$ \\
\hline $\mathrm{LSD}_{0.05}$ & 0.591 & 0.329 \\
\hline
\end{tabular}

Within the same column, various superscript letters indicate significant differences (Duncan, $P<0.05$ ). 


\section{Histopathological Results:}

Liver:

Microscopically, examination of liver of rats from G1 (untreated, control) revealed the normal histological structure of hepatic lobules. Also no histopathological changes were noticed in liver of rats from G2 which treated with GSE (fig. 1). Conversely, the examined sections from $\mathrm{G} 3$ which treated with $1 \mathrm{~g} / \mathrm{L} \mathrm{NaNO}{ }_{2}$ showed focal hepatic necrosis associated with inflammatory cells infiltration (fig. 2), kupffer cells activation and sinusoidal leucocytosis (fig. 3). While the liver of rats from $\mathrm{G} 4$ which treated with $2 \mathrm{~g} / \mathrm{L} \mathrm{NaNO} \mathrm{Na}_{2}$ showed dilatation and congestion of hepatic sinusoids (fig. 4), portal infiltration with mononuclear cells, focal hepatic necrosis associated with mononuclear cells infiltration (fig. 5), cytoplasmic vacuolization of hepatocytes and necrosis of sporadic hepatocytes (fig. 6). Meanwhile, some examined sections of liver of rats from G5 which treated with $1 \mathrm{~g} / \mathrm{L}$ $\mathrm{NaNO}_{2}$ and GSE showed kupffer cells activation (fig. 7), while other sections from same group revealed no histological changes (fig. 8). Moreover, liver of rats from G6 which treated with $2 \mathrm{~g} / \mathrm{L} \mathrm{NaNO}_{2}$ and GSE showed cytoplasmic vacuolization of hepatocytes (fig. 9).

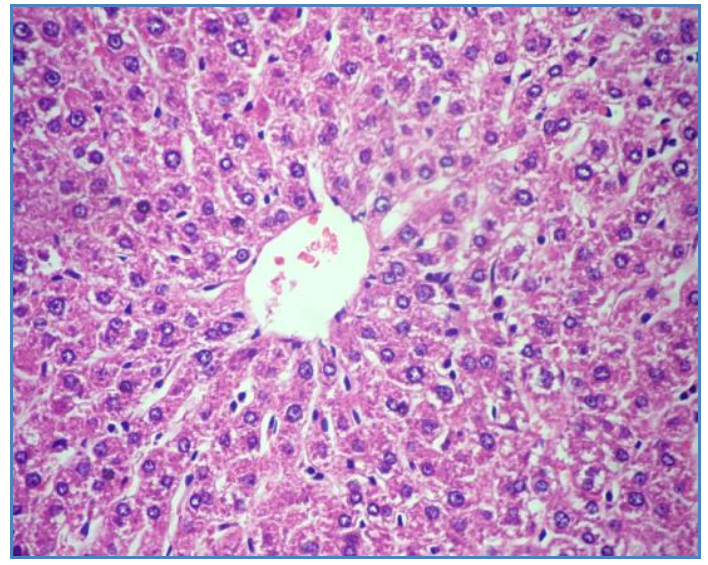

Fig. (1): Liver of rat from G2 showing the normal histological structure of hepatic lobule (H and E X 400).

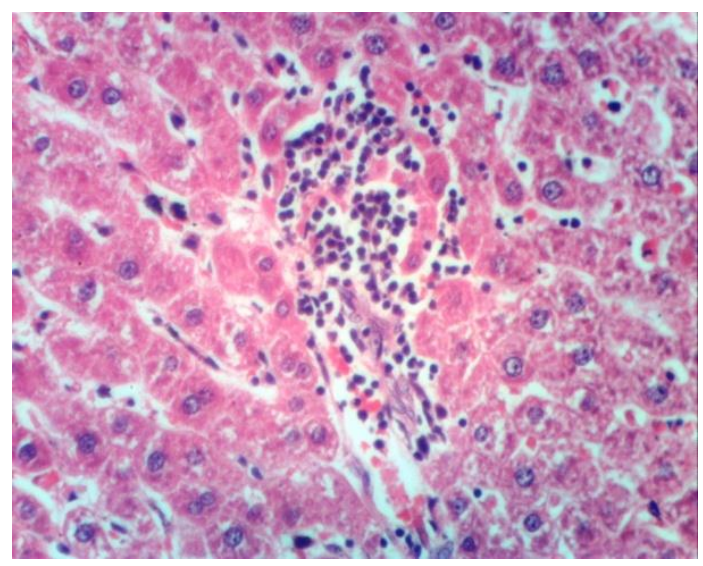

Fig. (3): Liver of rat from G3 showing kupffer cells activation and sinusoidal leucocytosis $(\mathrm{H}$ and $\mathrm{E}$ X 400).

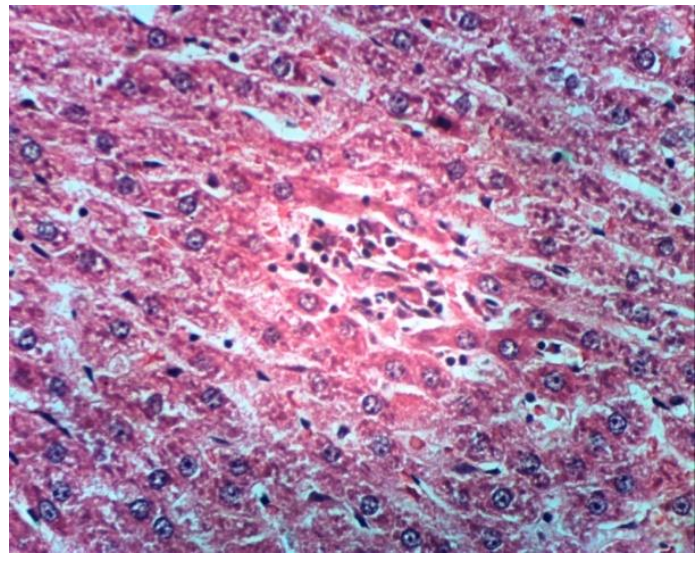

Fig. (2): Liver of rat from G3 showing focal hepatic necrosis associated with inflammatory cells infiltration (H and E X 400).

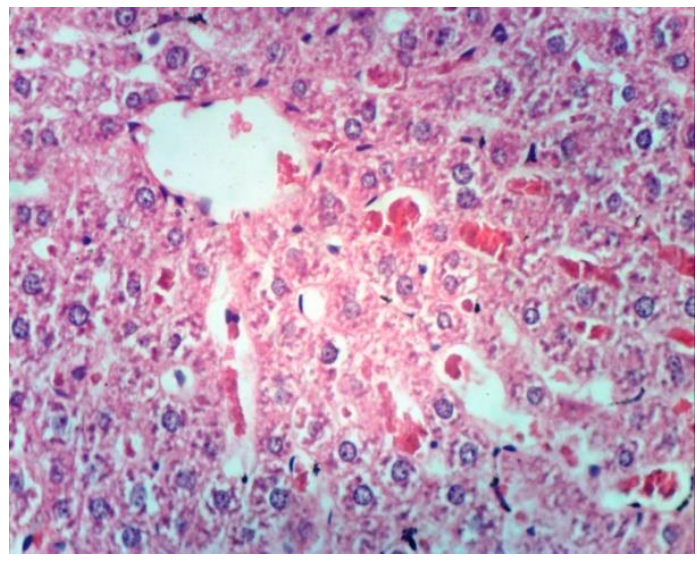

Fig. (4): Liver of rat from G4 showing dilatation and congestion of hepatic sinusoids ( $\mathrm{H}$ and $\mathrm{E} \mathrm{X}$ 400). 


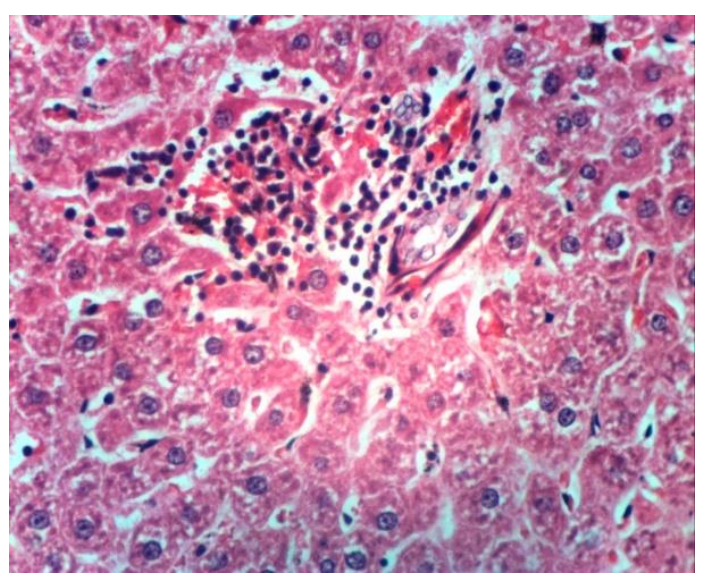

Fig. (5): Liver of rat from G4 showing portal infiltration with mononuclear cells, focal hepatic necrosis associated with mononuclear cells infiltration $(\mathrm{H}$ and E X 400).

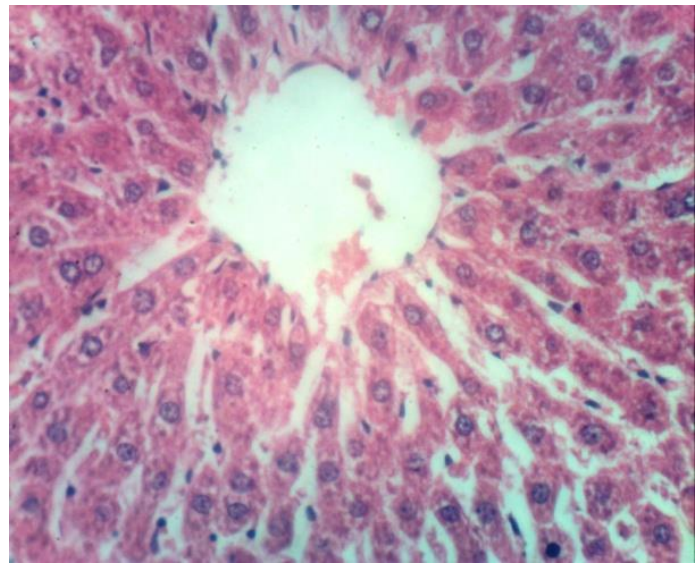

Fig. (7): Liver of rat from G5 showing kupffer cells activation $(\mathrm{H}$ and $\mathrm{E}$ X 400).

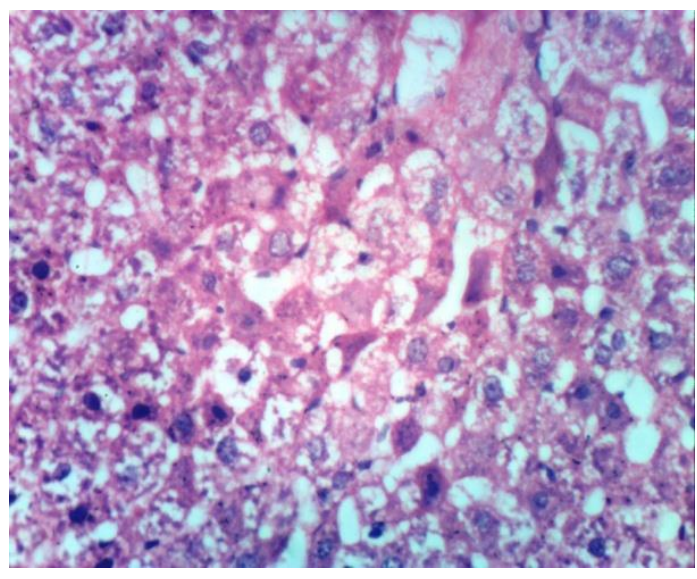

Fig. (6): Liver of rat from G4 showing cytoplasmic vacuolization of hepatocytes and necrosis of sporadic hepatocytes (H and E X 400).

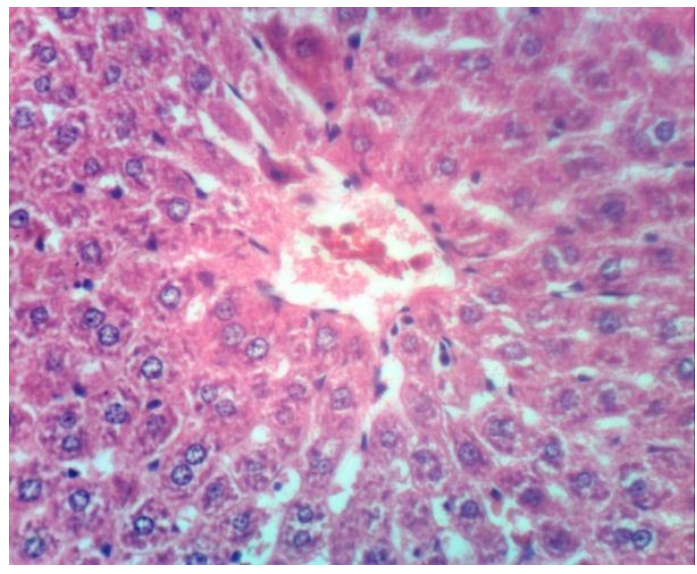

Fig. (8): Liver of rat from G5 showing apparent normal hepatocyte $(\mathrm{H}$ and E X 400).

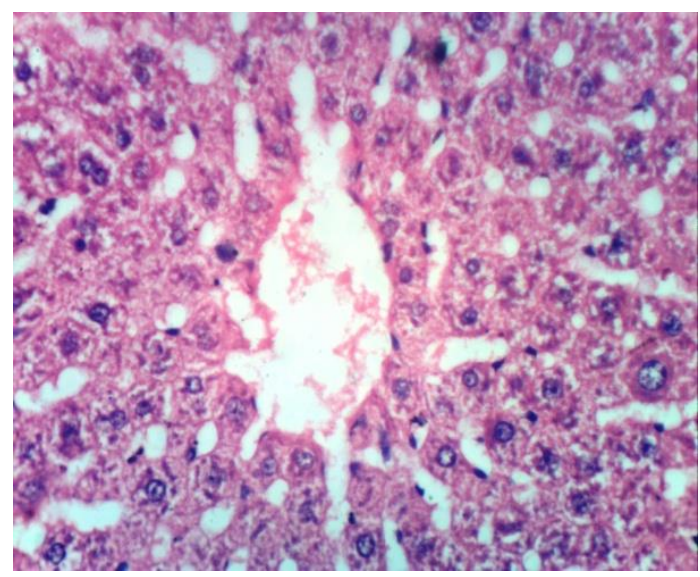

Fig. (9): Liver of rat from G6 showing cytoplasmic vacuolization of hepatocytes (H and E X 400). 


\section{Kidney:}

Microscopically, kidneys of control, untreated rats (G1) and rats administrated GSE (G2) revealed the normal histological structure of renal parenchyma (fig. 10). However, kidneys of rats from $\mathrm{G} 3$ which treated with $1 \mathrm{~g} / \mathrm{L} \mathrm{NaNO}_{2}$ revealed vacuolization of endothelial lining glomerular tuft with presence of protein cast in the lumen of renal tubules (fig. 11), hypertrophy of glumerular tuft (figs. 12 and 13), periglomerular fibroblasts proliferation (fig. 12) and vacuolization and congestion of glumerular tuft (fig. 13). Also, kidneys of rats from $\mathrm{G} 4$ which treated with $2 \mathrm{~g} / \mathrm{L} \mathrm{NaNO}{ }_{2}$ revealed eosinophilic protein cast in the lumen of renal tubules (fig. 14), hypertrophy and congestion of glumerular tuft, perivascular edema (fig. 15) and atrophy of glumerular tuft (fig. 16). No histological changes were found in kidneys of rats from G5 and G6 which co-treated with 1 and $2 \mathrm{~g} / \mathrm{L} \mathrm{NaNO}_{2}$ (respectively) and GSE (figs. 17 and 18).

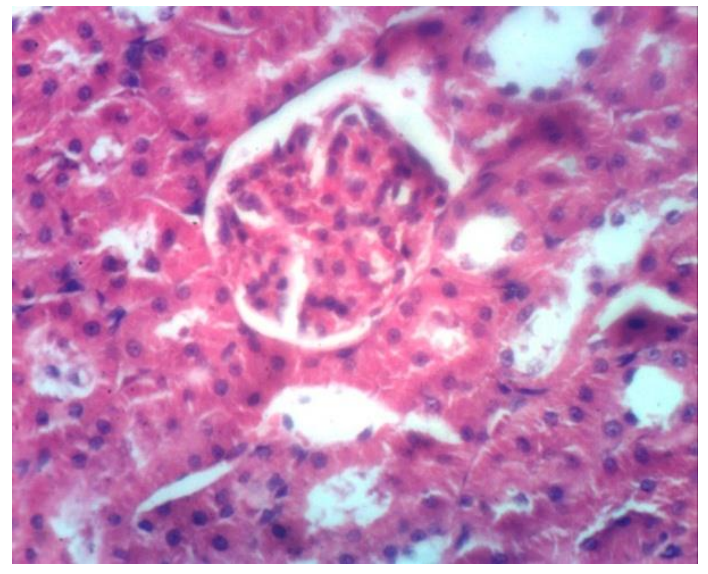

Fig. (10): Kidney of rat from G2 showing the normal histological structure of renal parenchyma $(\mathrm{H}$ and $\mathrm{E}$ X 400).

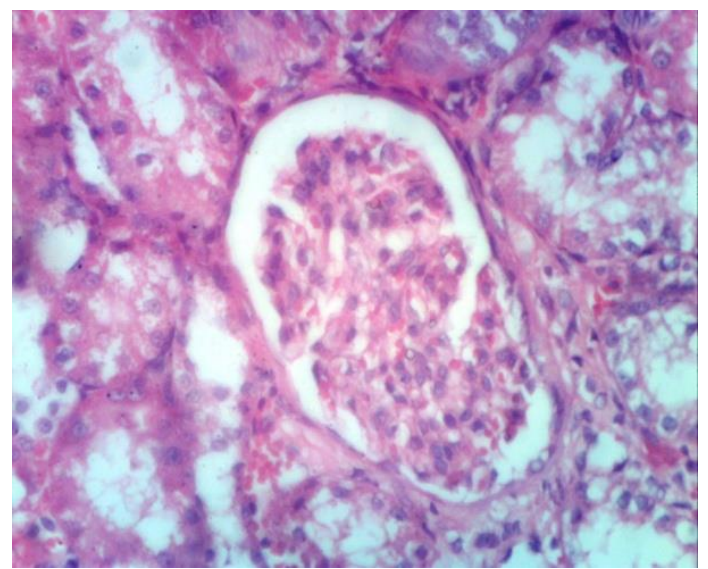

Fig. (12): Kidney of rat from G3 showing hypertrophy of glumerular tuft and periglomerular fibroblasts proliferation (H and E X 400).

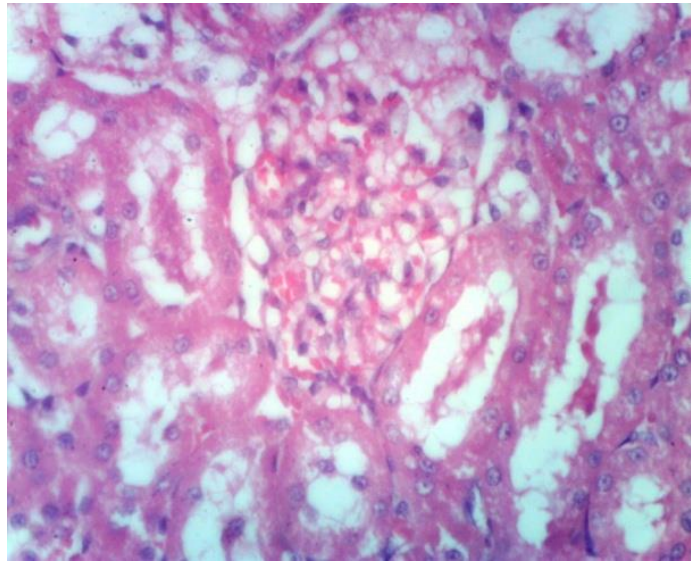

Fig. (11): Kidney of rat from G3 showing vacuolization of endothelial lining glomerular tuft with presence of protein cast in the lumen of renal tubules $(\mathrm{H}$ and $\mathrm{E}$ $\mathrm{X} 400$ )

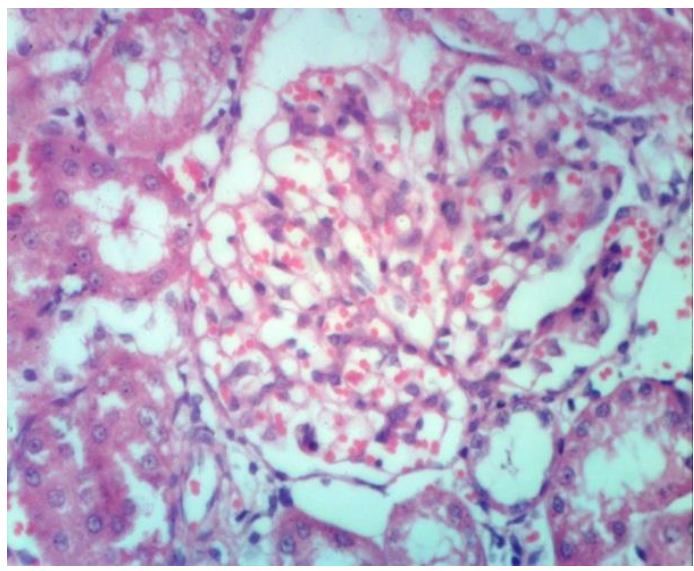

Fig. (13): Kidney of rat from G3 showing hypertrophy, vacuolization and congestion of glumerular tuft $(\mathrm{H}$ and $\mathrm{E} X$ 400). 


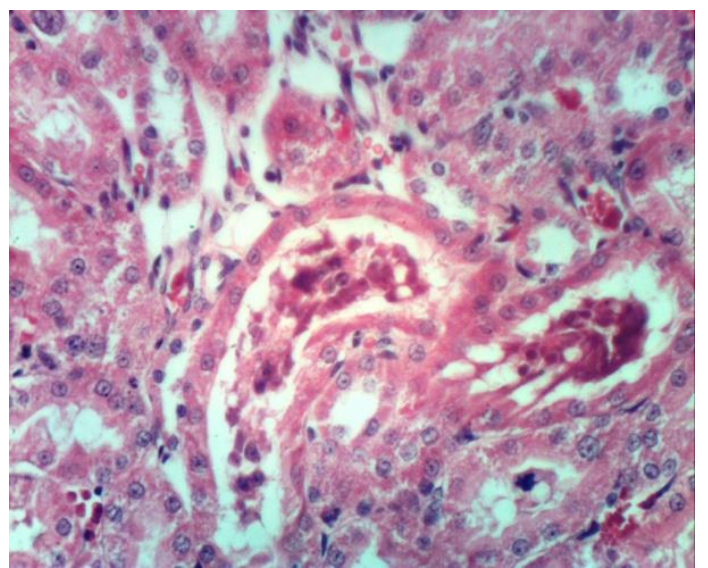

Fig. (14): Kidney of rat from G4 showing eosinophilic protein cast in the lumen of renal tubules ( $\mathrm{H}$ and $\mathrm{E}$ $X$ 400).

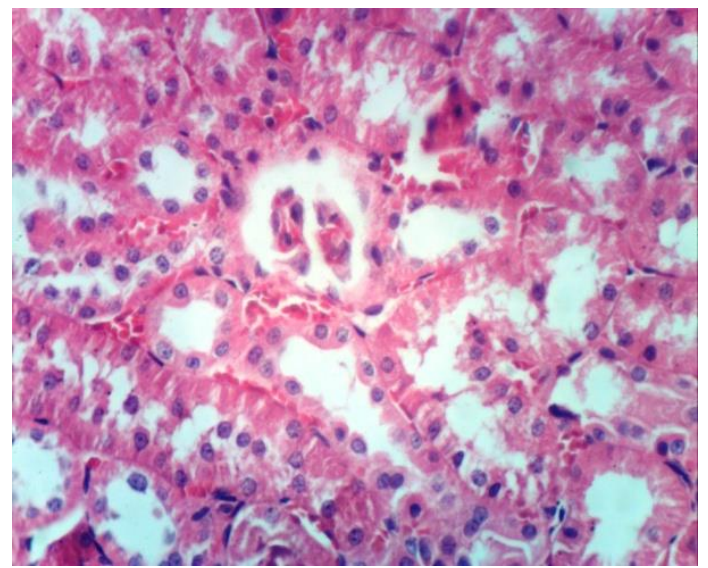

Fig. (16): Kidney of rat from G4 showing atrophy of glomerular tuft $(\mathrm{H}$ and E X 400).

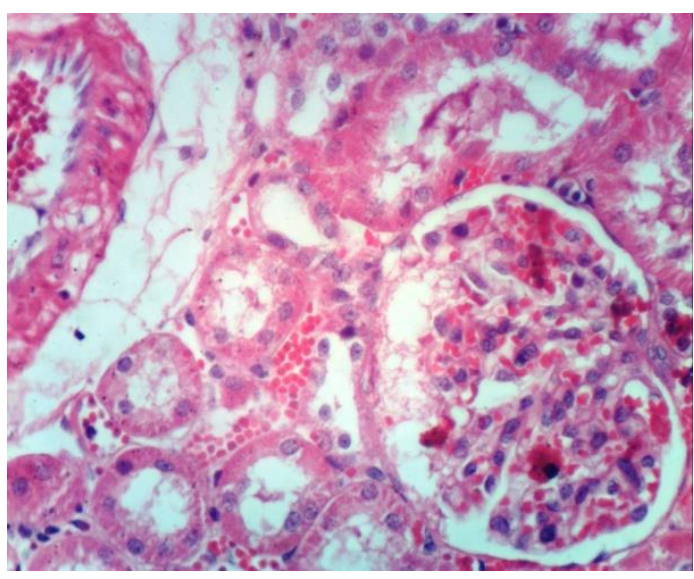

Fig. (15): Kidney of rat from G4 showing hypertrophy and congestion of glumerular tuft, perivascular edema ( $\mathrm{H}$ and $\mathrm{E} \mathrm{X}$ 400).

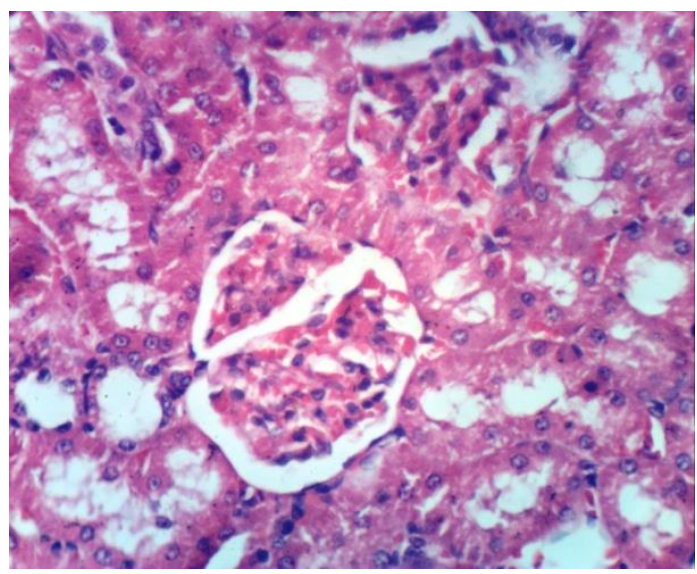

Fig. (17): kidney of rat from G5 showing apparent normal renal parenchyma (H and E X 400).

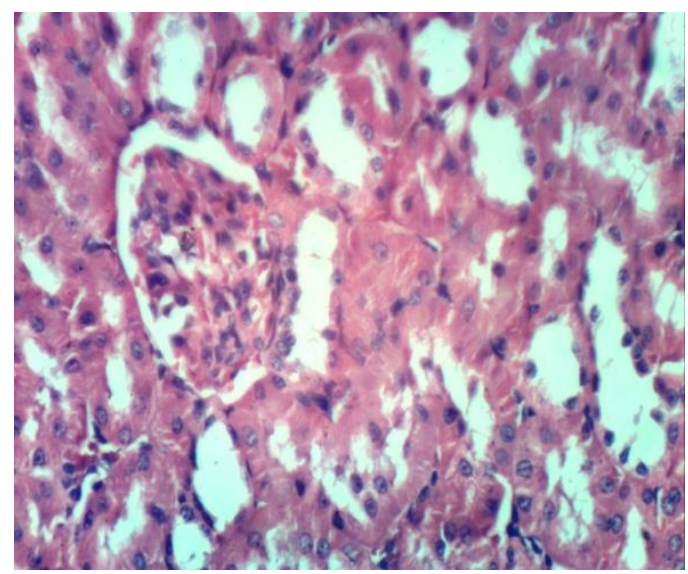

Fig. (18): Kidney of rat from G6 showing apparent normal renal parenchyma (H and E X 400). 


\section{Brain:}

Examination of brain of control, untreated rats (G1) and GSE-administrated rats (G2) revealed normal histological structure (fig. 19). Conversely, brain of rats from G3 which treated with $1 \mathrm{~g} / \mathrm{L} \mathrm{NaNO}_{2}$ showed pyknosis of neurons, neuronophagia (fig. 20) and menengial hemorrhage associated with inflammatory cells infiltration (fig. 21). Moreover, brain of rats from $\mathrm{G} 4$ which treated with $2 \mathrm{~g} / \mathrm{L} \mathrm{NaNO}{ }_{2}$ showed focal hemorrhage (fig. 22), hemorrhage in Virchow space (fig. 23), necrosis of neurons (fig. 24) and congestion of cerebral blood vessels (fig. 25). Meanwhile, brain of rats from G5 which administrated with $1 \mathrm{~g} / \mathrm{L} \mathrm{NaNO}{ }_{2}$ and GSE showed pyknosis of neurons (fig. 26). While, brain of rats from G6 which administrated with $2 \mathrm{~g} / \mathrm{L} \mathrm{NaNO}_{2}$ and GSE showed neuronophagia of pyknotic neurons (fig. 27).

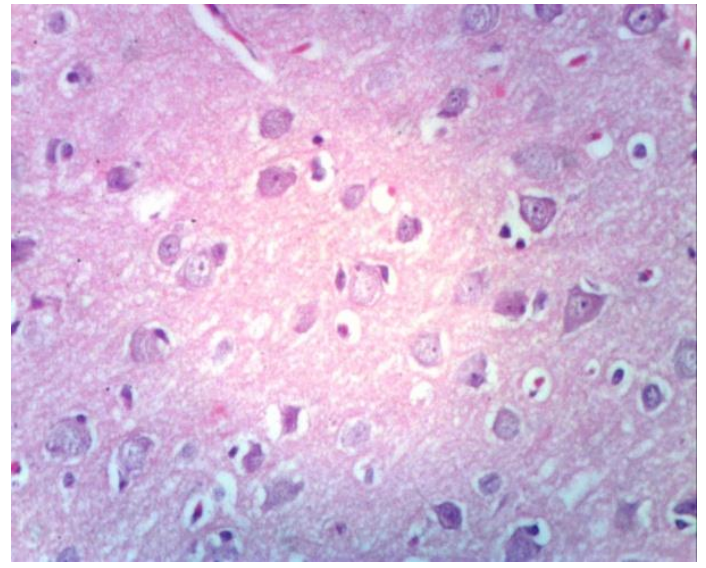

Fig. (19): Brain of rat from $\mathrm{G} 2$ showing the normal histological structure $(\mathrm{H}$ and E X 400).

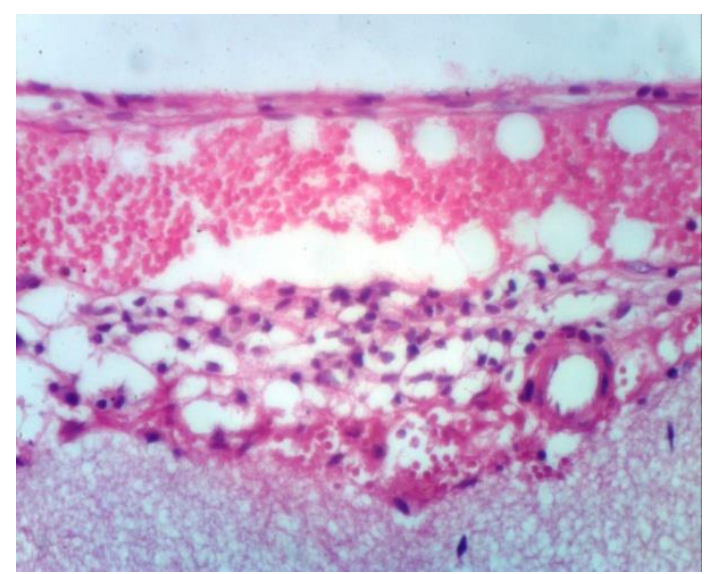

Fig. (21): Brain of rat from G3 showing menengial hemorrhage associated with inflammatory cells infiltration $(\mathrm{H}$ and $\mathrm{E} \mathrm{X}$ 400).

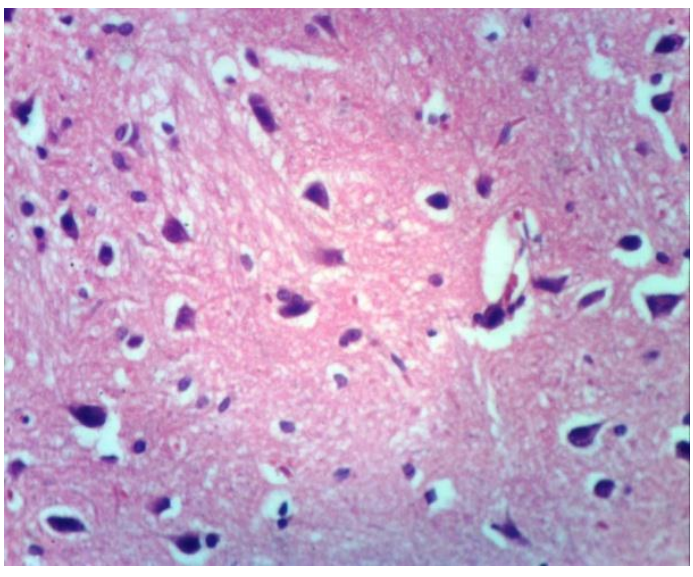

Fig. (20): Brain of rat from G3 showing pyknosis of neurons, neuronophagia $(\mathrm{H}$ and $\mathrm{E} \mathrm{X}$ 400).

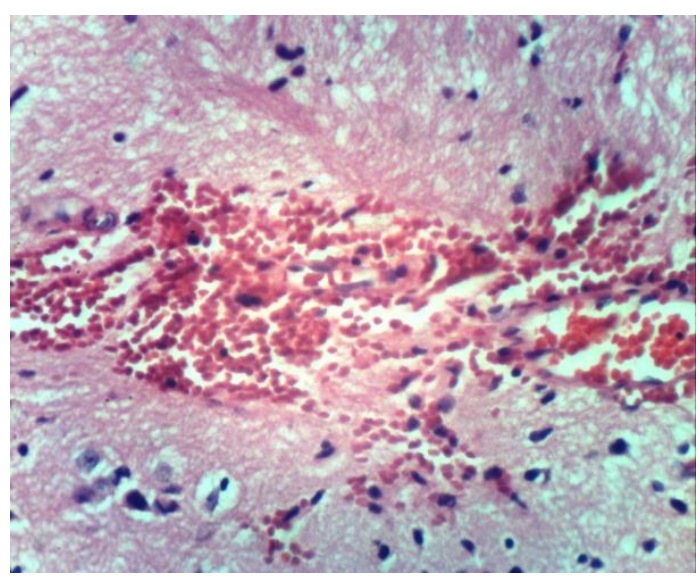

Fig. (22): Brain of rat from G4 showing focal hemorrhage $(\mathrm{H}$ and $\mathrm{E} \mathrm{X}$ 400). 


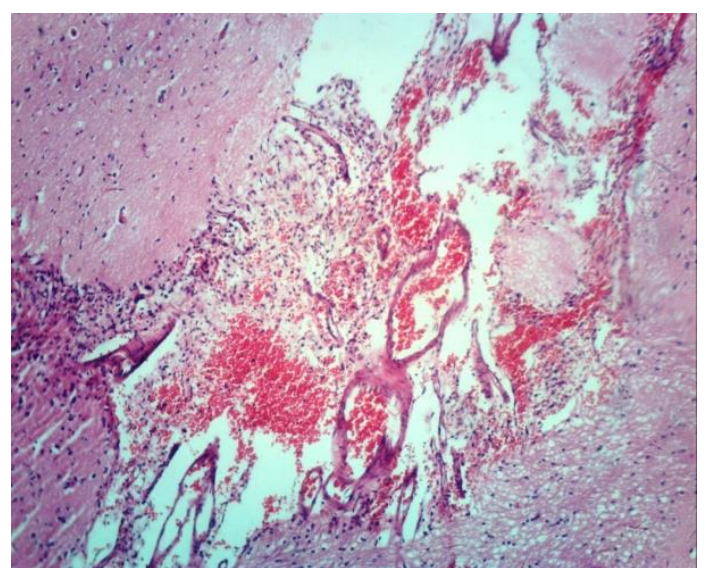

Fig. (23): Brain of rat from G4 showing hemorrhage in Virchow space $(\mathrm{H}$ and $\mathrm{E} \mathrm{X} \mathrm{400).}$

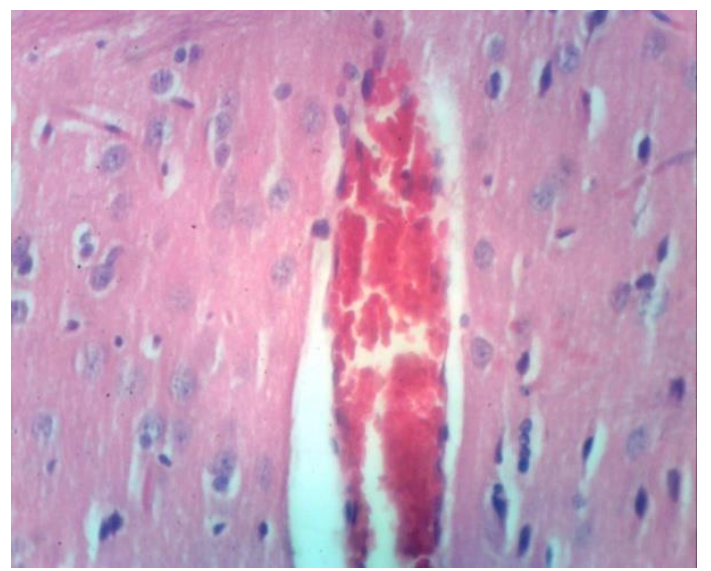

Fig. (25): Brain of rat from G4 showing congestion of cerebral blood vessels ( $\mathrm{H}$ and $\mathrm{E} \mathrm{X} \mathrm{400).}$

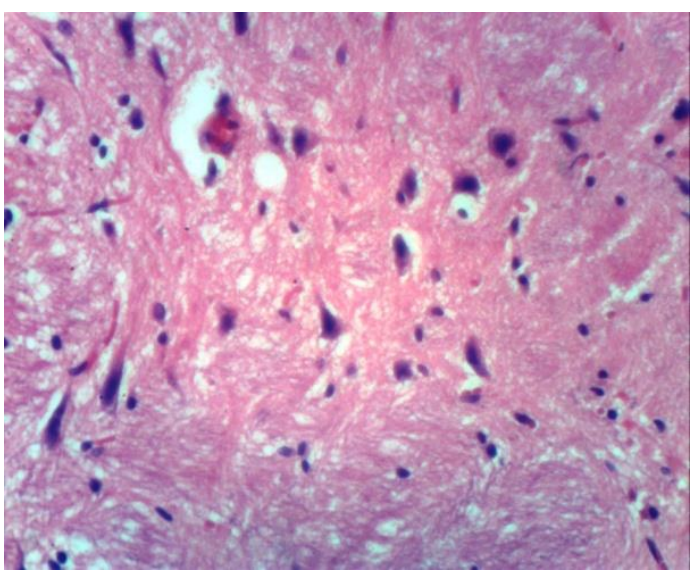

Fig. (24): Brain of rat from G4 necrosis of neurons (H and E X 400).

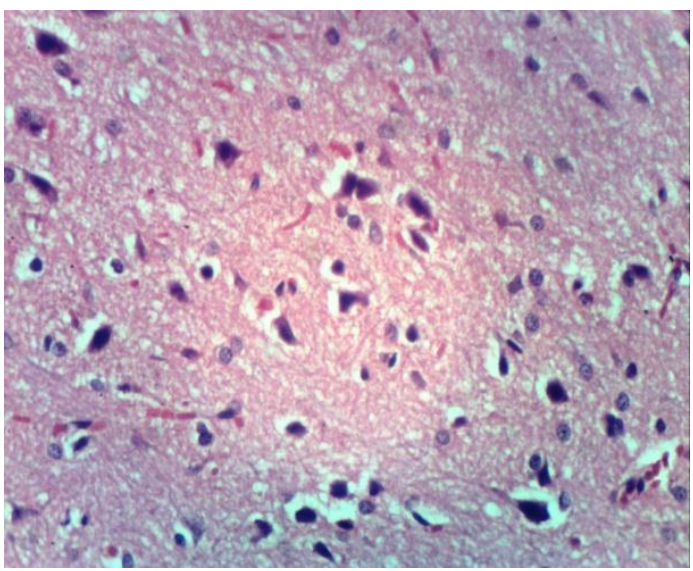

Fig. (26): Brain of rat from G5 showing pyknosis of neurons ( $\mathrm{H}$ and $\mathrm{E} \mathrm{X}$ 400).

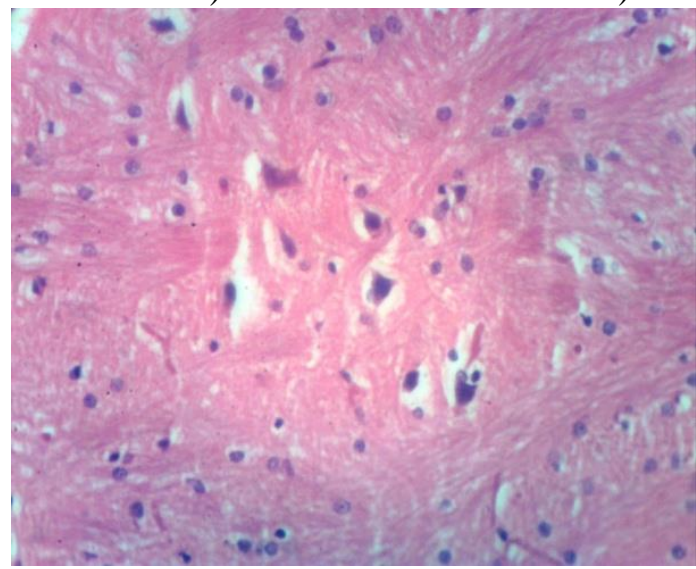

Fig. (27): Brain of rat from G6 showing neuronophagia of pyknotic neurons (H and E X 400).

\section{Heart:}

Histopathological examination of rats from G1 (control) and G2 (GSE-treated rats) revealed normal cardiac myocytes (fig. 28). However, heart of rats from G3 (treated with $1 \mathrm{~g} / \mathrm{L} \mathrm{NaNO}_{2}$ ) showed congestion of myocardial blood vessels (fig. 29) and myolysis of focal myocytes (fig. 30). Also, examined heart from $\mathrm{G} 4$ which treated with $2 \mathrm{~g} / \mathrm{L} \mathrm{NaNO} \mathrm{Na}_{2}$ showed 
congestion of myocardial blood vessels (fig. 31) and zenker's necrosis of sporsdic myocytes (fig. 32). No histological changes were noticed in heart of rats from G5 which treated with $1 \mathrm{~g} / \mathrm{L} \mathrm{NaNO}{ }_{2}$ and GSE (fig. 33). While, heart of rats from G6 which treated with $2 \mathrm{~g} / \mathrm{L} \mathrm{NaNO}$ and GSE showed congestion of myocardial blood vessels (fig. 34).

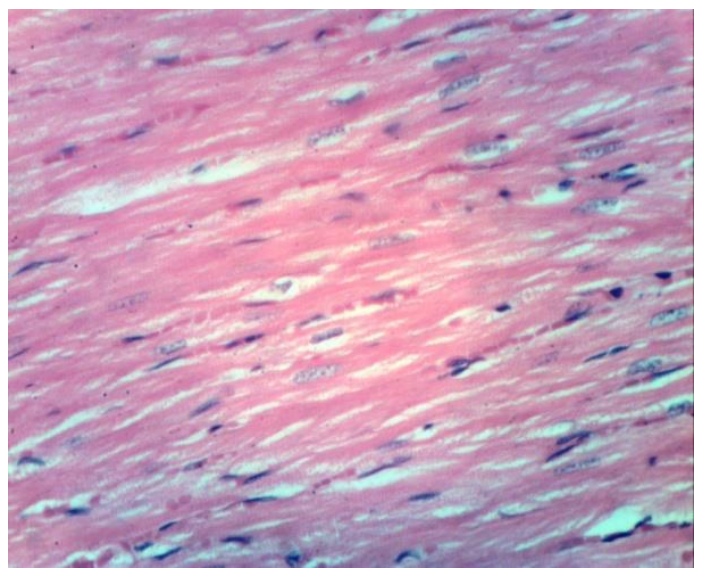

Fig. (28): Heart of rat from G2 showing the normal cardiac myocytes $(\mathrm{H}$ and E X 400).

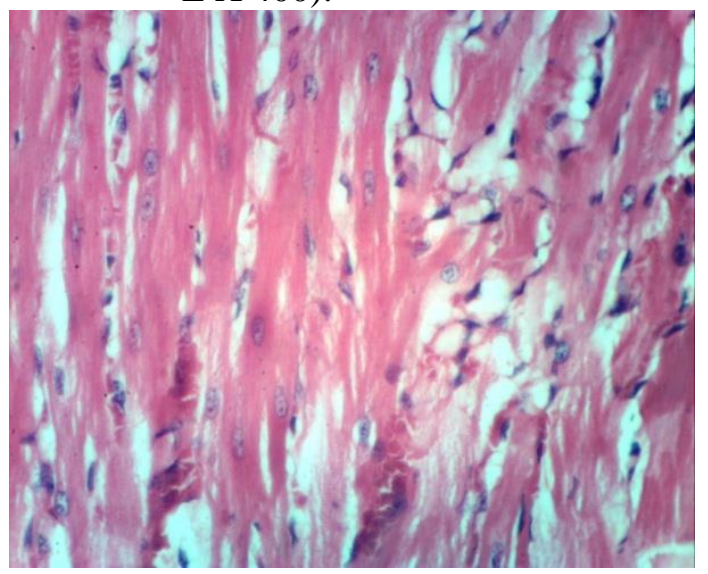

Fig. (30): Heart of rat from G3 showing myolysis of focal myocytes $(\mathrm{H}$ and $\mathrm{E} \mathrm{X} \mathrm{400).}$

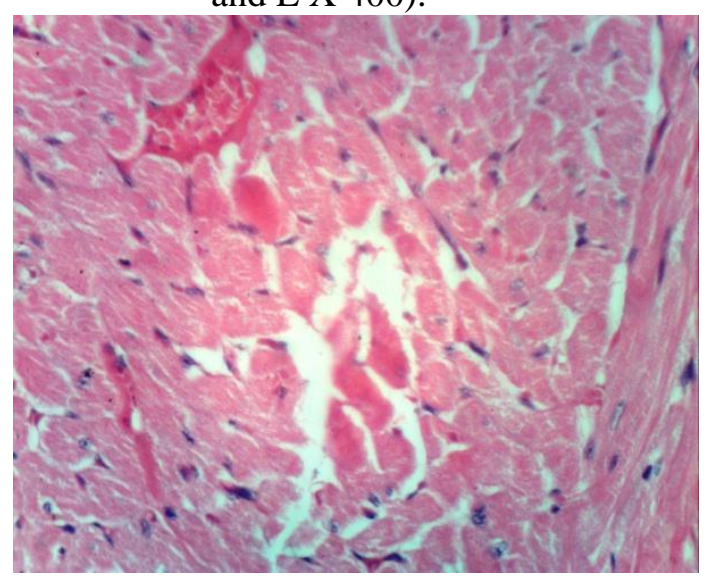

Fig. (32): Heart of rat from G4 showing zenker's necrosis of sporsdic myocytes (H and E X 400).

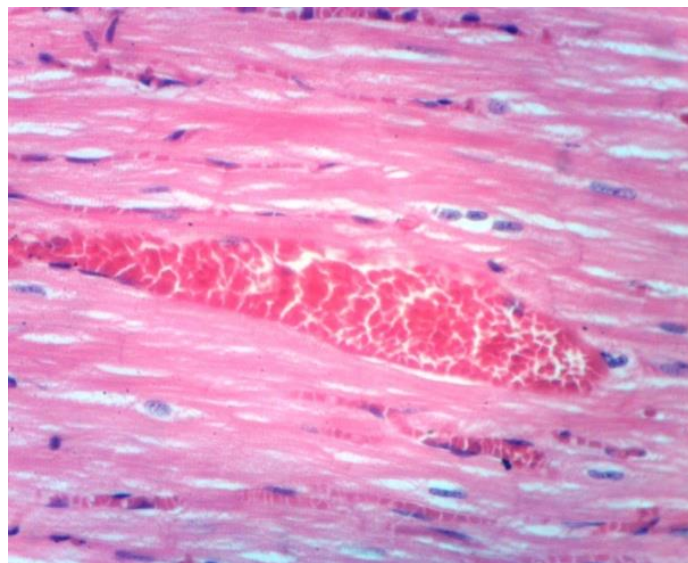

Fig. (29): Heart of rat from G3 showing congestion of myocardial blood vessels ( $\mathrm{H}$ and $\mathrm{E} X \mathrm{X} 400$ ).

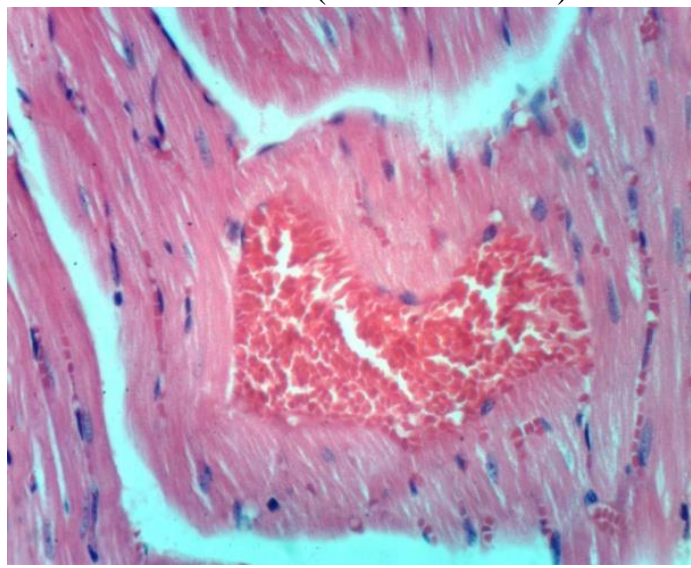

Fig. (31): Heart of rat from G4 showing congestion of myocardial blood vessels ( $\mathrm{H}$ and $\mathrm{E} \mathrm{X} \mathrm{400).}$

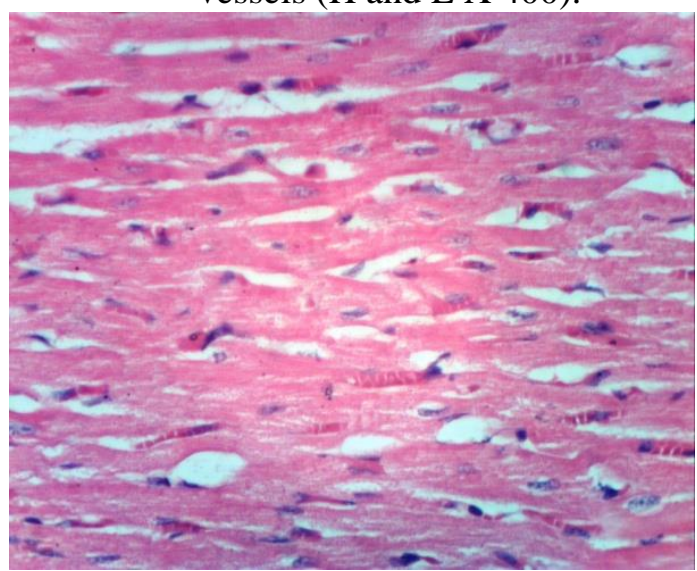

Fig. (33): Heart of rat from G5 normal cardiac myocytes $(\mathrm{H}$ and $\mathrm{E} \mathrm{X}$ 400). 


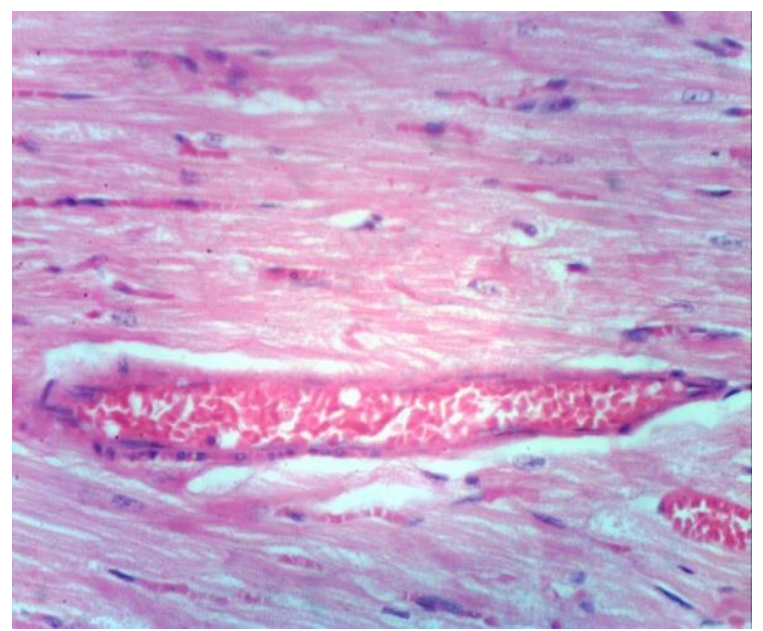

Fig. (34): Heart of rat from G6 showing congestion of myocardial blood vessels ( $\mathrm{H}$ and $\mathrm{E} \mathrm{X} \mathrm{400).}$

\section{Lungs}

Microscopically, lungs of control, untreated rats (G1) and GSE-treated rats (G2) showed normal histological structure (fig. 35). Conversely, examined lungs of rats from G3 (treated with $1 \mathrm{~g} / \mathrm{L} \mathrm{NaNO}_{2}$ ) showed atelectasis (fig. 36) and bronchitis (fig. 37). The examined lungs of rats from $\mathrm{G} 4$ (treated with $2 \mathrm{~g} / \mathrm{L} \quad \mathrm{NaNO}_{2}$ ) showed perivascular inflammatory cells infiltration (fig. 38), interstitial pneumonia (fig. 39) and pulmonary hemorrhage (fig. 40). While, no histological changes were noticed in lungs of rats from G5 (treated with $1 \mathrm{~g} / \mathrm{L} \mathrm{NaNO}{ }_{2}$ and GSE) and in some examined sections of lungs of rats from G6 (treated with $2 \mathrm{~g} / \mathrm{L} \mathrm{NaNO}_{2}$ and GSE) (fig. 41), whereas, other sections showed focal interstitial pneumonia (fig. 42).

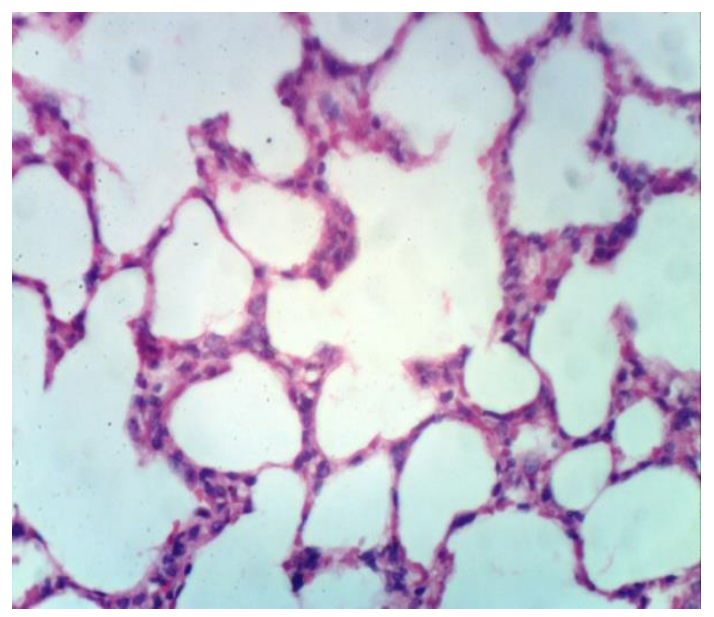

Fig. (35): Lung of rat from $\mathrm{G} 2$ showing the normal histological structure $(\mathrm{H}$ and E X 400).

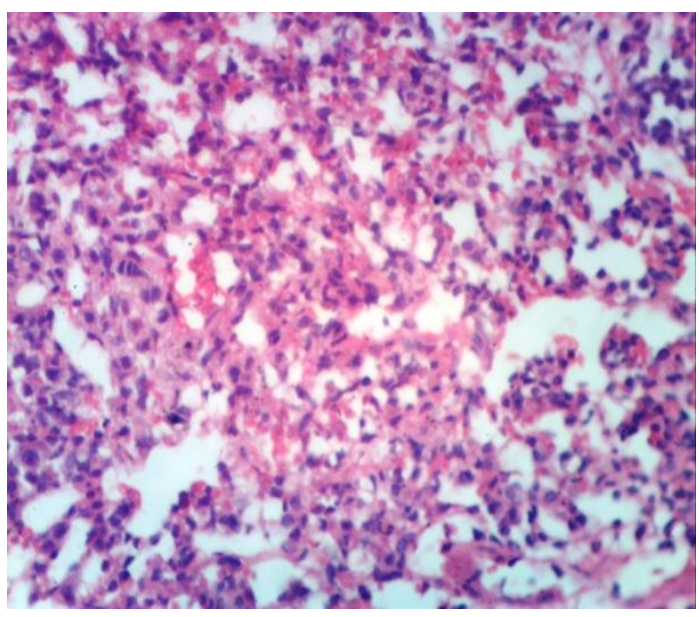

Fig. (36): Lung of rat from G3 showing atelectasis ( $\mathrm{H}$ and $\mathrm{E} \mathrm{X} \mathrm{400).}$ 


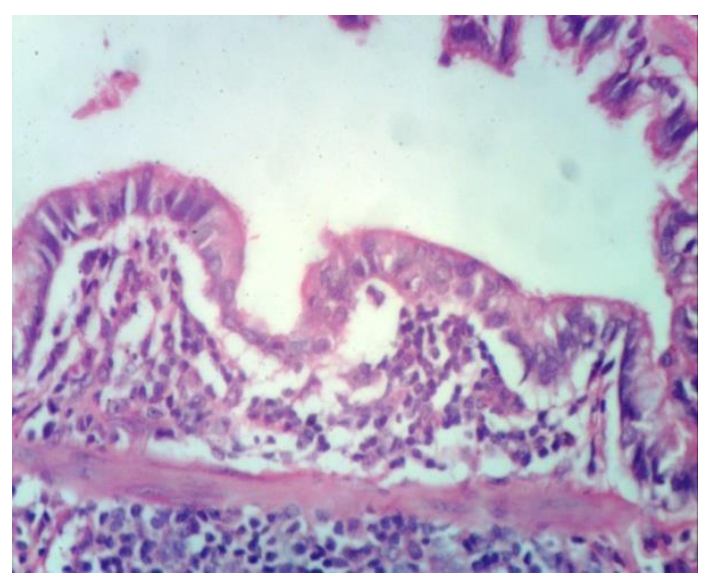

Fig. (37): Lung of rat from G3 showing bronchitis. Note mononuclear cells infiltration in bronchial mucosa (H and E X 400).

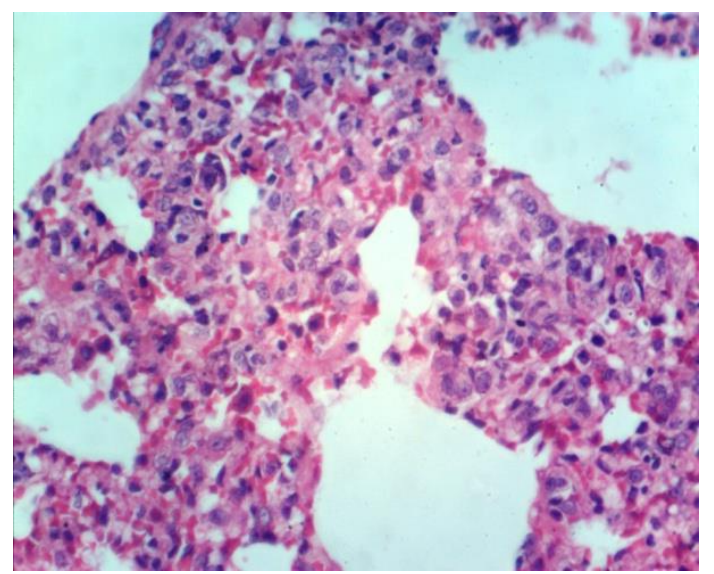

Fig. (39): Lung of rat from G4 showing interstitial pneumonia $(\mathrm{H}$ and $\mathrm{E}$ X 400).

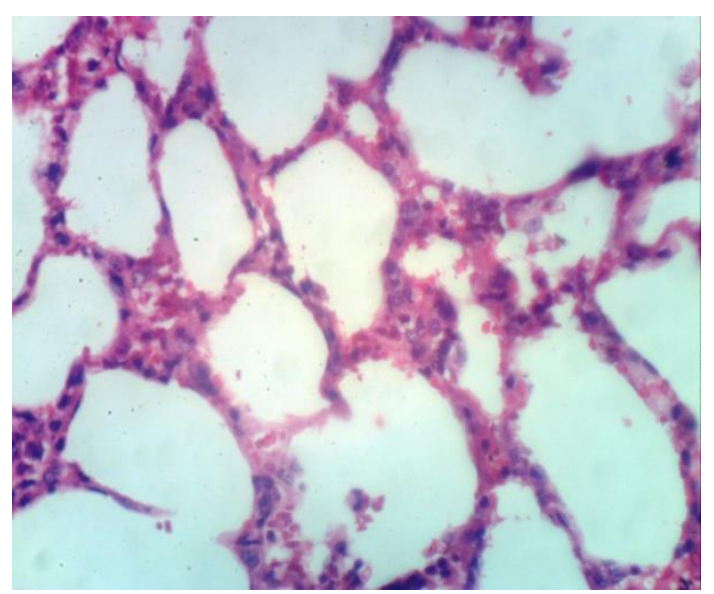

Fig. (41): Lung of rat from G5 showing no histopathological changes ( $\mathrm{H}$ and E X 400).

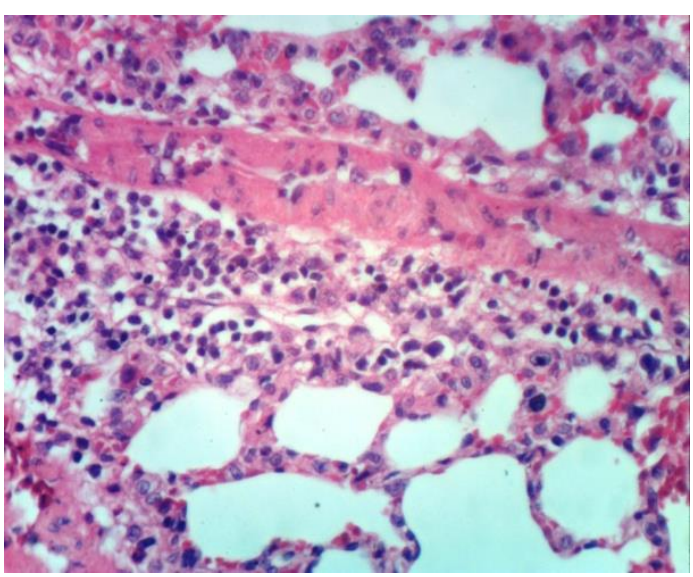

Fig. (38): Lung of rat from G4 showing perivascular inflammatory cells infiltration (H and E X 400).

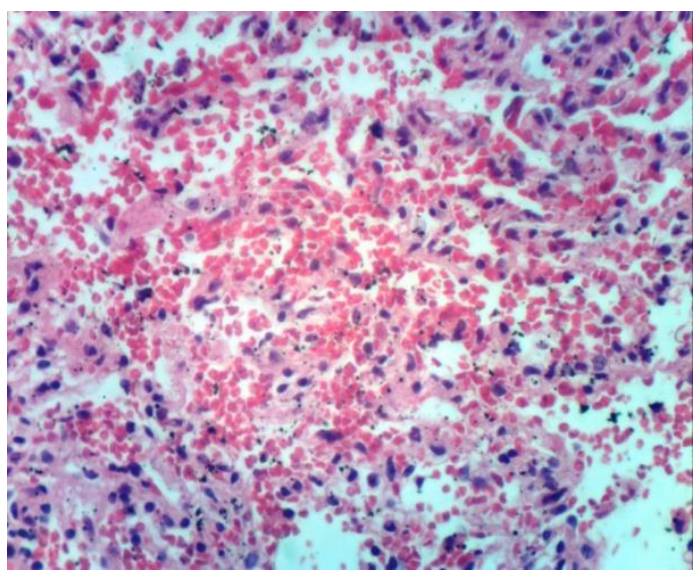

Fig. (40): Lung of rat from G4 pulmonary hemorrhage (H and E X 400).

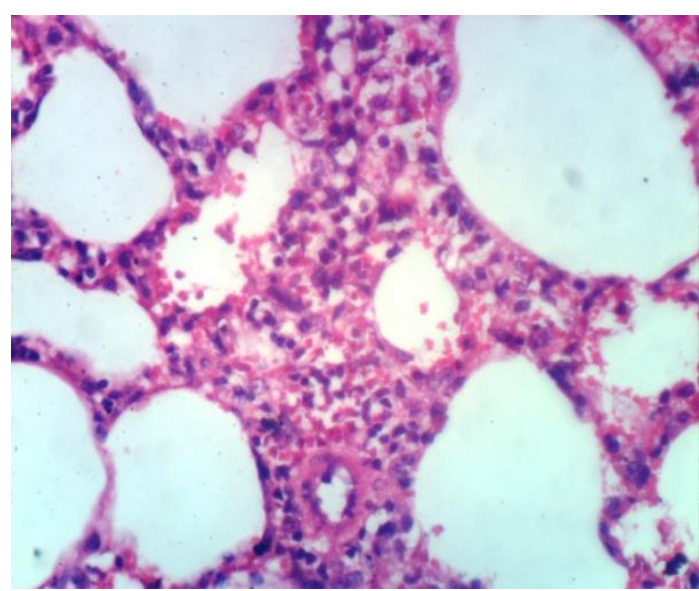

Fig. (42): Lung of rat from G6 focal interstitial pneumonia $(\mathrm{H}$ and $\mathrm{E}$ X 400). 


\section{Testes:}

Microscopically, examination of testes of rats from G1 (control, untreated) and rats from G2 (GSE-treated rats) showed normal seminiferous tubules and complete spermatogenesis (fig. 43). However, testes of rats from G3 which treated with $1 \mathrm{~g} / \mathrm{L} \mathrm{NaNO}{ }_{2}$ revealed degeneration of spermatogoneal cells lining seminiferous tubules and necrosis leydig cells (fig. 44). Also, examination of testes of rats from G4 which treated with $2 \mathrm{~g} / \mathrm{L}$ $\mathrm{NaNO}_{2}$ revealed interstitial edema (fig. 45) and degeneration of spermatogoneal cells lining seminiferous tubules (fig. 46). Meanwhile, testes of rats from G5 and G6 which treated with 1and $2 \mathrm{~g} / \mathrm{L} \mathrm{NaNO} \mathrm{Na}_{2}$ respectively in combination with GSE revealed no histological changes (figs. 47 and 48).

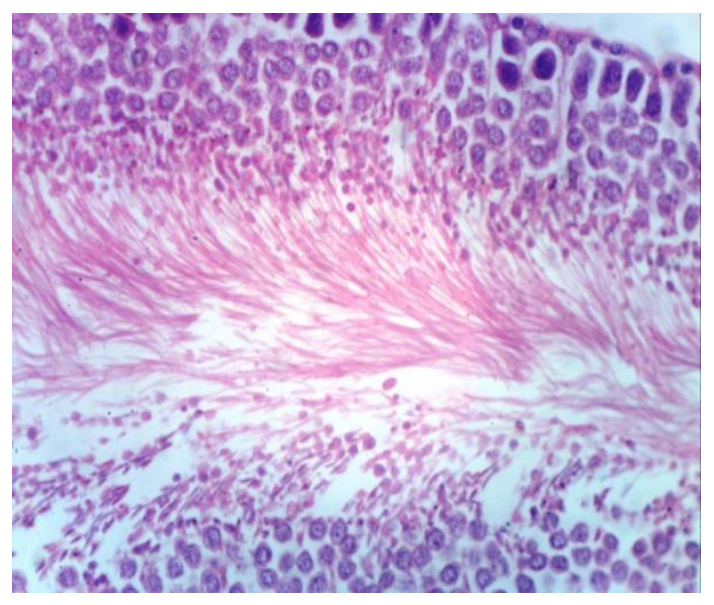

Fig. (43): Testis of rat from G2 showing the normal seminiferous tubules and complete spermatogenesis (H and E X 400).

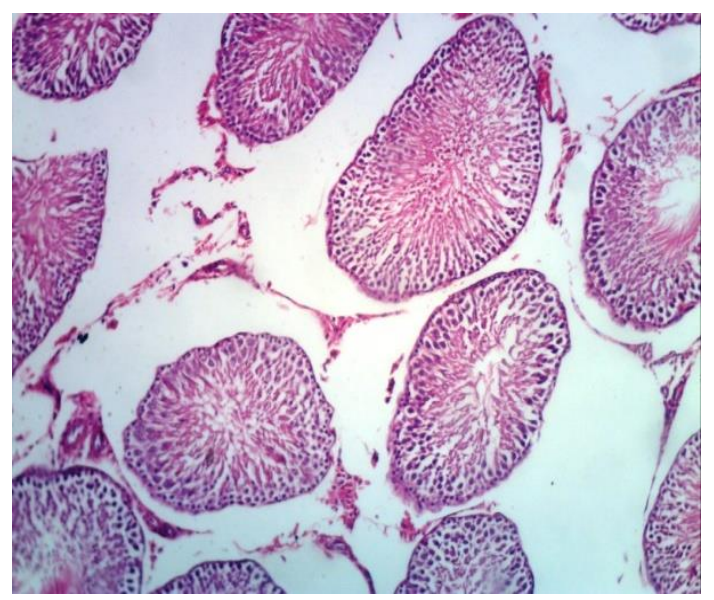

Fig. (45): Testis of rat from G4 showing interstitial edema $(\mathrm{H}$ and $\mathrm{E} \mathrm{X}$ 200).

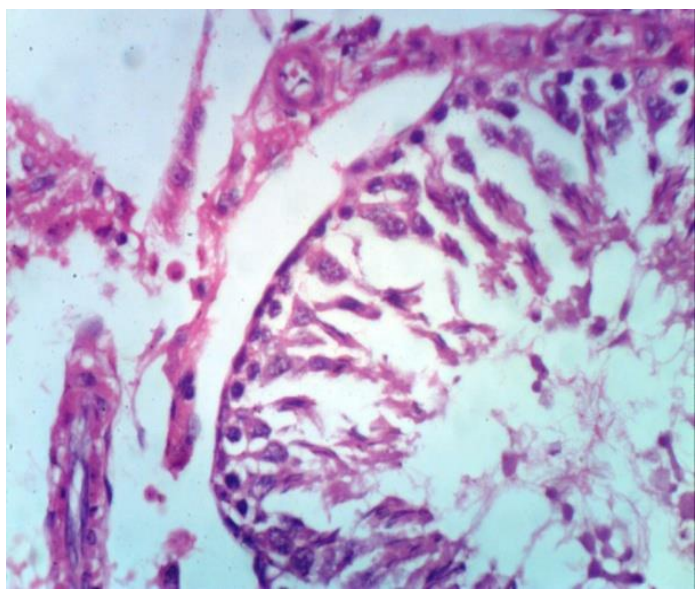

Fig. (44): Testis of rat from G3 showing degeneration of spermatogoneal cells lining seminiferous tubules and necrosis leydig cells ( $\mathrm{H}$ and E X 400).

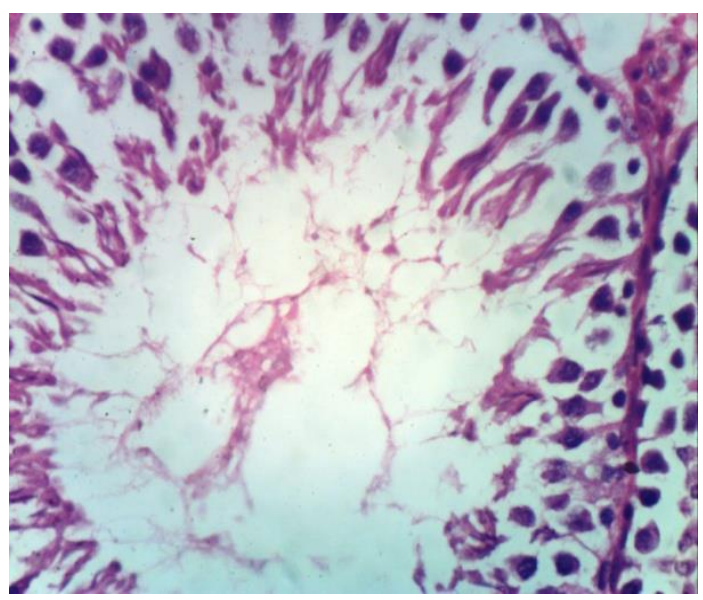

Fig. (46): Testis of rat from G4 showing degeneration of spermatogoneal cells lining seminiferous tubules (H and E X 400). 


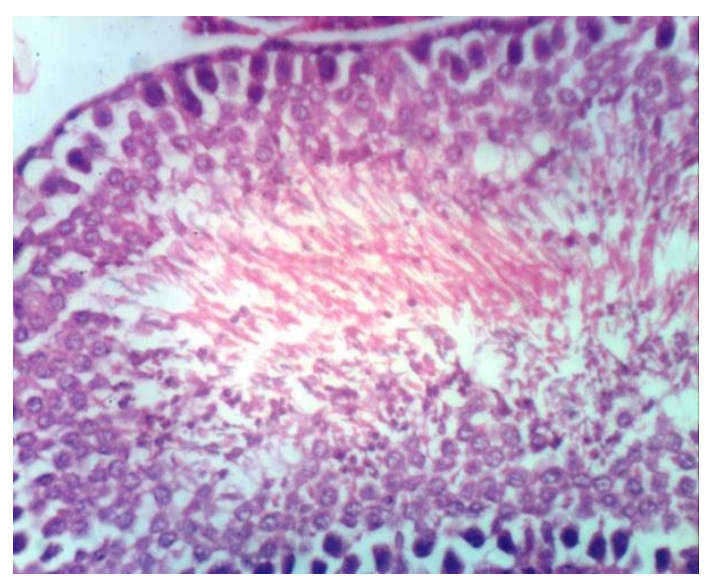

Fig. (47): Testis of rat from G5 showing normal seminiferous tubules and complete spermatogenesis $(\mathrm{H}$ and $\mathrm{E} X \mathrm{X} 400)$.

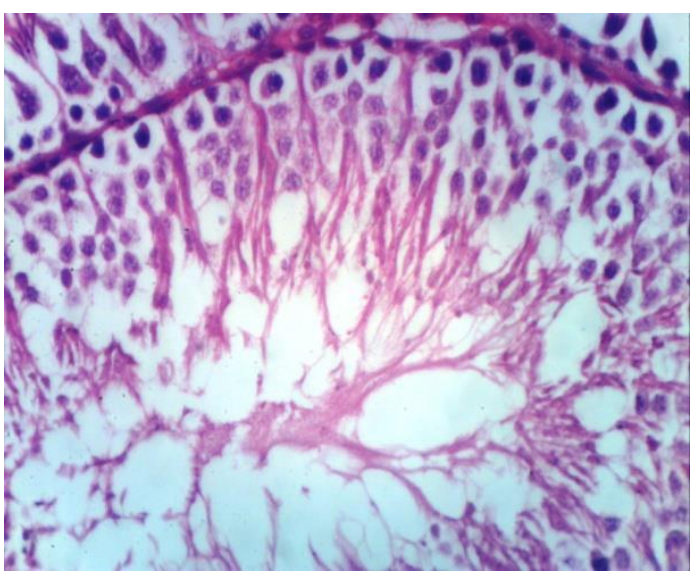

Fig. (48): Testis of rat from G6 showing no histological changes $(\mathrm{H}$ and $\mathrm{E} \mathrm{X}$ 400).

\section{DISCUSSION}

$\mathrm{NaNO}_{2}$ is a main preservative in cured meat products, fish and some types of cheese and occurs naturally in many foods, particularly vegetables (Dejam et al., 2005). However, toxicity to humans and animals is well documented in nitrite overexposure (RCHAS, 2000 and WHO, 2007). The $\mathrm{NaNO}_{2}$ and other additives may react with amines of the foods in the stomach and produce nitrosamines and free radicals. Such products may increase lipid peroxidation, which can be harmful to different organs including liver and kidney (Choi et al., 2002). On the other hand, many plant-derived drugs used in modern medicine are developed by ethnomedical leads and subsequent ethnopharmacological studies. Plants containing flavonoids have been reported to possess strong antioxidant properties (Raj and Shalini, 1999). The antioxidant activity of GSE make it candidate for addition to food and beverages to retard deterioration; it is possible that the antioxidant activity of GSE ingested with these foods would also support physiological defenses against in vivo-generated free radicals species (Ricardo-DaSilva et al., 1991 and Bentivegna and Whitney, 2002).

The data of this study have shown that $\mathrm{NaNO}_{2}$ administration at dose level of $1 \mathrm{~g}$ and $2 \mathrm{~g} / \mathrm{L}$ in drinking water for 4 and 8 weeks resulted in significant dose- dependent decrease of blood WBCs, RBCs count and $\mathrm{Hb}$ concentration. These results are in agreement with the finding of Helal and Elsaid (2006) showed significantly decrease of RBCs\%, WBCs\%, $\mathrm{Ht} \%$ and $\mathrm{Hb} \%$ in rats received $\mathrm{NaNO}_{2}(10 \mathrm{mg} / \mathrm{kg} /$ day $)$ orally for 30days. Moreover, Gluhcheva et al. (2012) stated that acute treatment of rats with $\mathrm{NaNO}_{2}$ induces significant hematological and rheological changes therefore monitoring of these parameters is necessary when exposed to high doses or prolonged treatment with the compound. The decrease of $\mathrm{RBCs}$ count and $\mathrm{Hb}$ concentration in our study was associated with increase of $\mathrm{MetHb}$ formation in blood of $\mathrm{NaNO}_{2}$-intoxicated rats. Such increase was noted by Imaizumi $\boldsymbol{e t}$ al . (1980) who reported that maximum levels of MetHb $(45-80 \%)$ were reached one hour after dosing Sprague-Dawley rats with $150 \mathrm{mg} / \mathrm{kg} \mathrm{B.W} \mathrm{NaNO}$. Also, In Sprague-Dawley rats receiving a single dose of $30 \mathrm{mg} / \mathrm{kg} \mathrm{B} . \mathrm{W}$ of $\mathrm{NaNO}_{2}$ in aqueous solution by gavage $(10-15 \%$ of $\mathrm{LD}_{50}$ ), plasma nitrite and MetHb levels were increased after 2.8 minutes and maximum effects (plasma nitrite $=15 \%$, MetHb $=12 \%$ ) were observed after 22.5 minutes (Hirneth and Classen, 1984). The experimental model of Kohn et al. (2002) predicts that $10 \%$ of the $\mathrm{Hb}$ is oxidized to the ferric form after oral doses of $15.9 \mathrm{mg} / \mathrm{kg} \mathrm{NaNO} \mathrm{N}_{2}$ in male rats and 11.0 $\mathrm{mg} / \mathrm{kg} \mathrm{NaNO} \mathrm{N}_{2}$ in female rats and after intravenous doses of 8.9 and $7.1 \mathrm{mg} / \mathrm{kg}$ in male and female rats, respectively. The chemical reactivity of $\mathrm{NaNO}_{2}$ with $\mathrm{Hb}$ may enhance heme- or 
iron-mediated toxicities. Nitrite is known to cause free radical generation, as it can stimulate oxidation of ferrous ions in oxyhemoglobin to form MetHb as well as various ROS (reactive oxygen species) (Gladwin et al., 2004 and Baky et al., 2010). The nitrite ion, its metabolites, and lipid peroxidation products are supposed to react with sulfhydryl groups of the lipid bilayer and protein components of erythrocyte membrane and change its structure (Maeda et al., 1987). Nitrite-promoted $\mathrm{Ca}^{2+}$ influx in blood cells activates phospholipases, which increase the proportion of phospholipids with a rigid structure in the membrane (Kaya and Miura, 1982). Moreover, WBCs decrease is associated with the failure of the hematopoietic tissues to produce new WBC (Tan et al., 1992). It is suggested that the decrease in $\mathrm{RBCs}$ and $\mathrm{Hb}$ concentration may be attributed to microcytic and/or hypochromic anemia possibly as a consequence of the toxic effect of $\mathrm{NaNO}_{2}$ on bone marrow, spleen and liver (Aboel-Zahab et al., 1997).

This study has shown that GSE alone does not have any negative impact on hematological parameters and MetHb formation. In the current study, the decrease in hematological parameters and increase in MetHb formation was reversed near normalcy by the administration of GSE in all experimental periods, moreover RBCs and MetHb were return to normal at end of experiment in $1 \mathrm{~g} \mathrm{NaNO}$-treated rats. Similarly, no significant changes in hematological parameters in male and female Sprague-Dawley rats provided with GSE in the diet at levels of $0.5,1.0$, or $2.0 \%$, for a period of 90 days (Wren et al., 2002) and in male and female rats provided with GSE in the diet at levels of $0.63,1.25$ or $2.5 \%$, for a period of 3 months (Bentivegna and Whitney, 2002). While the relative improvement of these parameters may be due to suggested antioxidant activity of GSE in vivo includes stimulating enzyme production of nitric oxide, oxygen radical scavenging and inhibition of nitrositive stress (Bagchi et al., 2000 and Roychowdhury et al., 2001).

The current study revealed that $\mathrm{NaNO}_{2}$ induced dose-dependent liver damage as evidenced by changes in liver function parameters and histopathological examination. The administration of $\mathrm{NaNO}_{2}$ for 8 weeks induced dose-dependent increase in ALT, AST, ALP and GGT activities and significant dose dependant-decrease in TP and albumin. Thus increased concentrations of these enzymes in serums normally indicate hepatocellular damage and leakage of these enzymes from liver cells into the blood stream (Carey, 2000). Similar to our results, Helal and Elsaid (2006) reported increased activities of ALT, AST, ALP and GGT and decrease concentrations of TP and albumin in $\mathrm{NaNO}_{2}$-treated rats. Also, Krishnamoorthy and Sangeetha (2008), Hassan et al. (2009) and Efuruibe et al. (2013) reported increase of ALT, AST, GGT and ALP activities in rats as a result of treatment with $\mathrm{NaNO}_{2}(300 \mathrm{mg} / \mathrm{kg} \mathrm{B.W}$ for 30 days, $80 \mathrm{mg} / \mathrm{kg}$ B.W for 3 months and single dose of $6.20 \mathrm{mg}$, respectively). El-Sheikh and Khalil (2011) fed rats with $0.2 \% \mathrm{NaNO}_{2}$ for 6 weeks and recorded decrease of TP and albumin. The increase in the activity of AST, ALT, GGT and ALP enzymes in the serum of $\mathrm{NaNO}_{2}$-treated rats could be attributed to the toxic effect of nitroso-compounds, formed in the acidic environment of the stomach, in causing severe hepatic necrosis Hassan et al. (2009). However, the indicated inhibitory effect of $\mathrm{NaNO}_{2}$ on the biosynthesis of protein could be attributed to stimulation of the thyroid and the adrenal glands by $\mathrm{NaNO}_{2}$ which can lead to a blockage in protein synthesis, fast breakdown, and increased rate of free amino acids, and decreased protein turnover (Eremin and Yocharina, 1981). In addition, nitrite interactions results into nitric oxide release, which can inhibit total protein synthesis (Kolpakov et al., 1995). The severity of hepatic lesions in our study was found to be parallel to $\mathrm{NaNO}_{2}$ concentration in water. The liver of $1 \mathrm{~g} / \mathrm{L} \mathrm{NaNO}_{2}$-treated rats showed focal hepatic necrosis associated with inflammatory cells infiltration, kupffer cells activation and sinusoidal leucocytosis while the liver of $2 \mathrm{~g} / \mathrm{L} \mathrm{NaNO}_{2}$-treated rats showed the same previous lesions besides dilatation and congestion of hepatic sinusoids, portal infiltration with mononuclear cells, cytoplasmic vacuolization of hepatocytes and necrosis of 
sporadic hepatocytes. Such hepatic damage by $\mathrm{NaNO}_{2}$ was previously recorded by Gomaa and Abd Elaziz (2011) in rats by $80 \mathrm{mg} / \mathrm{kg} \mathrm{NaNO}{ }_{2}$ oral administration for 2 months. Also, Abebe et al. (2013) recorded severe haemorrhage within the sinusoid and the portal triad was infiltrated by inflammatory cells in liver of $\mathrm{NaNO}_{2}$-treated rats $(25$ and $50 \mathrm{mg} / \mathrm{kg})$. While, Efuruibe et al. (2013) were reported mild periportal cellular infiltration as a result of $\mathrm{NaNO}_{2}$ administration in rats. Such liver damage could be explained by formation of free radicals $\left(\mathrm{ONOO}^{-}\right)$from nitric oxide. Both $\mathrm{NO}$ and oxygen radicals could react further to produce other oxidant and nitro compounds such as peroxynitrite to induce liver injury (Liaudet et al., 2000 and Chow and Hong 2002) and it play an important role in death of liver cells (Krishnamoorthy and Sangeetha, 2008).

This study has shown that GSE alone does not have any negative impact on liver function parameters and histology of liver. As revealed previously in toxicological studies on GSE by Wren et al. (2002) and Bentivegna and Whitney (2002). The current results revealed that co-treatment with GSE and $\mathrm{NaNO}_{2}$ resulted in a significant improvement in all liver function parameters and liver histology as indicated by significant alleviation of toxic effect of $\mathrm{NaNO}_{2}$ on ALT, AST, ALP, TP and albumin levels in serum. Especially with low $\mathrm{NaNO}_{2}$ dose and at $8^{\text {th }}$ week of treatment, but it failed to normalize them except GGT, which restored to normal. Moreover the hepatic structure of $1 \mathrm{~g} \mathrm{NaNO}_{2}$ and GSE-treated rats was reversed to normal. The protective effect of GSE on liver against oxidative damage was previously recorded by Dulundu et al. (2007) who reported that GSE administration was significantly able to decrease the increase of AST and ALT levels as a result of biliary obstruction induced by bile duct legation in serum of rats due to its antioxidant and antifibrotic prosperities. Chis et al. (2009) suggested that the GSE enhanced the antioxidant defense against reactive oxygen species produced under hyperglycaemic conditions, hence protecting the liver cells. Yousef $\boldsymbol{e t}$ al. (2009) reported that GSE antagonized cisplatininduced increase in the activities of AST and ALT and decrease concentrations of TP and albumin in rats. Furthermore, El-Beshbishy et al. (2010) observed that oral intake of GSE to tamoxifen-intoxicated rats resulted in attenuation of histopathological changes in liver and corrected all liver function parameters (ALT, AST, ALP and GGT). More interesting Hassan (2012) noted that the ethanol grape seed extract (EGSE) was more effective than water grape seed extract (WGSE) against hepatotoxicity of alcohol in rats. Hepatoprotective activity of the grape seed extracts could be attributed to the antioxidant effect of the constituents and enhanced antioxidant defenses.

Kidneys represent the major control system maintaining body homeostasis. The serum concentration of urea and creatinine determine renal function and are thus biomarkers for kidney disease (Levey et al., 1999). The present results revealed dose-dependent increase of creatinine and urea concentration in serum of $\mathrm{NaNO}_{2}$-intoxicated rats. Such finding was previously reported in rats by Hassan et al. (2009) and El-Sheikh and Khalil (2011). These effects could be attributed to the changes in the threshold of tubular re-absorption, renal blood flow and glomerular filtration rate (GFR) (Zurovsky and Haber, 1995). The elevation in kidney function biomarker was synchronized with observed damage in kidney. The noticed lesions in kidneys of both $\mathrm{NaNO}_{2}$-treated groups $(1 \mathrm{~g}$ and $2 \mathrm{~g} / \mathrm{L})$ showed the same degree of severity. Kidney of $\mathrm{NaNO}_{2}$-intoxicated groups revealed vacuolization of endothelial lining glomerular tuft with presence of protein cast in the lumen of renal tubules, hypertrophy of glumerular tuft, periglomerular fibroblasts proliferation, vacuolization and congestion of glumerular tuft, perivascular edema and atrophy of glumerular tuft. Such kidney damage by $\mathrm{NaNO}_{2}$ was previously recorded by Gomaa and Abd Elaziz (2011) and Abebe et al. (2013).

This study has shown that GSE alone does not have any negative impact on kidney function parameters and histology of kidney. As revealed previously in toxicological studies 
on GSE by Wren et al. (2002) and Bentivegna and Whitney (2002). The current results revealed that co-treatment with GSE and $\mathrm{NaNO}_{2}$ resulted in a significant improvement in all kidney function parameters and Kidney histology which indicated by significant alleviation of toxic effect of $\mathrm{NaNO}_{2}$ on creatinine and urea levels in serum especially with low $\mathrm{NaNO}_{2}$ dose and at $8^{\text {th }}$ week of treatment. Both of them were restored to normalcy level at the end of experiment. Moreover the GSE administration reversed toxic effect of both $\mathrm{NaNO}_{2}$ dose and restore kidney histology to normal. The protective effect of GSE on kidney against oxidative damage was previously recorded by Yousef et al. (2009) and Saad et al. (2009) they reported that GSE antagonized cisplatin-induced increase in concentration of creatinine and urea in serum of rats and damage in Kidney genomic DNA. The mechanism underlying GSE nephroprotection may be due to the marked radical scavenging ability of GSE proanthocyanidins (Sato et al., 2005).

Lipids play an important role in cardiovascular disease, not only by way of hyperlipidaemia and the development of atherosclerosis, but also by modifying the composition, structure, and stability of cellular membranes. Excess lipids in the blood are considered to accelerate the development of arteriosclerosis and are the major risk factor in myocardial infarction. High levels of circulating cholesterol and its accumulation in heart tissue are well associated with cardiovascular damage (Salter and White, 1996). The results of the present study revealed time and dose-dependent increase in total cholesterol and triglycerides concentration in serum of $\mathrm{NaNO}_{2}$-treated rats. Such results have been provided by Helal and Elsaid (2006) in $\mathrm{NaNO}_{2}$-intoxicated rats. These biochemical changes were coincident with the histopathological changes in heart tissue induced by $\mathrm{NaNO}_{2}$ in the present study which represented by congestion of myocardial blood vessels and myolysis of focal myocytes in $1 \mathrm{~g} / \mathrm{L} \mathrm{NaNO}_{2}$-treated group. And congestion of myocardial blood vessels and zenker's necrosis of sporsdic myocytes in $2 \mathrm{~g} / \mathrm{L} \mathrm{NaNO}_{2}$-treated group. In an early chronic toxicity study (Gruener and Shuval, 1973), rats were given nitrite in the drinking water at doses equivalent to $0,10,100,200$ and $300 \mathrm{mg} / \mathrm{kg}$ B.W/day) corresponding to $0,6.7,67$, $124,201 \mathrm{mg} / \mathrm{kg}$ B.W per day for nitrite). At the highest dose, focal degeneration and fibrosis of the heart muscle as well as dilatation of coronary arteries were also observed. More recently Gomaa and Abd Elaziz (2011) reported necrosis of myocardial muscle cells in $\mathrm{NaNO}_{2}$-treated rats.

It worthy to note that GSE were had no negative effect on total cholesterol, triglycerides and heart tissue. The hypercholesterolemic, increased triglycerides and heart injury induced by $\mathrm{NaNO}_{2}$ in the present study were significantly alleviated by GSE administration. GSE administration restores cholesterol level at $4^{\text {th }}$ week of treatment, triglycerides levels along experimental period in serum and heart histology to normal of $1 \mathrm{~g}$ $\mathrm{NaNO}_{2}$-treated rats. These results are in agreement with finding of Karthikeyan $\boldsymbol{e t}$ al. (2007) showed that the administration of grape seed proanthocyanidins significantly maintained the cholesterol, phospholipids, triglycerides, and free fatty acids levels in serum and heart tissue of the isoproterenol -induced myocardial injury in rats. The experiments conclude that grape seed proanthocyanidins possess cardioprotective and hypolipidemic effect on the treatment of ISO-induced myocardial injury. Also, Yousef et al. (2009) reported that GSE antagonized cisplatin-induced increase in cholesterol and triglycerides. Furthermore Jiao et al. (2010) concluded that the hypocholesterolemic activity of grape seed proanthocyanidins was most likely mediated by enhancement of bile acid excretion and upregulation of cholesterol-7 $\alpha$-hydroxylase (CYP7A1). On the other hand, potential mechanisms by which grape polyphenols may exert cardioprotective effects include a reduction in oxidative stress, modulation of the inflammatory cascade, improvement in vascular endothelial function (eg, flow-mediated dilatation [FMD]), and protection against 
atherothrombotic episodes including myocardial ischemia and inhibition of platelet aggregation (Cui et al., 2002 Shanmuganayagam et al., 2002 and Delmas et al., 2005).

Our results clearly showed that there was a significant dose dependent-increase in serum glucose concentration of $\mathrm{NaNO}_{2}$-treated rats in all experimental periods. The same results was obtained by Hassan et al. (2009) and Gomaa and Abd Elaziz (2011) in rats and by Abdul-Ameer and Abed (2012) in mice. The findings suggest nitrite-stimulation of gluconeogenesis (Wiechetek et al., 1992), and glucose shift from tissue to blood or an impairment of glucose mobilization. Furthermore, nitroso-compounds can alter the antioxidant system causing disturbance in the metabolic processes leading to hyperglycemia (Anil et al., 2005).

However, in our study serum glucose levels were ameliorated upon GSE supplementation and return to normal level in serum of $1 \mathrm{~g} \mathrm{NaNO}_{2}$-treated rats at $8^{\text {th }}$ week. The hypoglycemic effect of grape seed proanthocyanidins was previously reported by ElAlfy et al. (2005) and Terra et al. (2009). The mechanism underlying such protection is mediated via prevention and restoration of pancreatic antioxidant defense systems and stimulating insulin release by pancreatic $\beta$-cells. An additional mechanism of the antihyperglycemic action of grape seed may be through stimulating the surviving pancreatic cells to release more insulin (El-Alfy $\boldsymbol{e t}$ al. 2005). A recent study by Pinent $\boldsymbol{e t}$ al. (2004) has also shown that grape-seed procyanidins possess an insulinomimetic activity on insulin sensitive cell lines.

Lipid peroxidation, a type of oxidative degeneration of polyunsaturated fatty acids (PUFA), has been linked with altered membrane structure and enzyme inactivation (Karthikeyan et al., 2007). Consequently, lipid peroxidation is known to induce cellular damage and is responsible for ROS induced organ damage. Lipid peroxidation of membranes is regulated by the availability of substrate in the form of PUFA, the availability of inducers, such as free radicals and excited state molecules, to initiate propagation, the antioxidant defense status of environment, and the physical status of the membrane lipids (Anandan et al., 1998). GSH is one of the essential compounds for regulation of variety of cell functions. It has a direct antioxidant function by reacting with superoxide radicals, peroxy radicals and singlet oxygen followed by the formation of oxidized glutathione (GSSG) and other disulfides. GST is GSH-dependent antioxidant enzymes (Karthikeyan et al., 2007). GST catalyses the conjugation of GSH via the sulfhydryl group, to electrophilic centers on a wide variety of substrates. This activity is useful in the detoxification of endogenous compounds such as peroxidised lipids (Valavanidisa et al., 2006). The enzyme SOD catalyzes dismutation of the superoxide anion $\left(\mathrm{O}^{-2}\right)$ into hydrogen peroxide $\left(\mathrm{H}_{2} \mathrm{O}_{2}\right)$, which is then detoxified to $\mathrm{H}_{2} \mathrm{O}$ by catalase (Mukherjee $\boldsymbol{e t}$ al., 2003).

Results of this study showed that $\mathrm{NaNO}_{2}$ induce oxidative stress in rats as evidenced by dose-dependent increase of serum MDA and dose-dependent decrease of plasma GSH, GST and SOD. Oxidative stress induced by $\mathrm{NaNO}_{2}$ was previously fully described by Krishnamoorthy and Sangeetha (2008); El-Sheikh and Khalil (2011) and Gomaa and Abd Elaziz (2011).

The present data indicated that treatment with GSE alone didn't alter any of oxidative stress parameters and significantly alleviated the toxic effect of $\mathrm{NaNO}_{2}$ on antioxidant defense system especially on lower dose and longer time as GSH and GST levels were restored to normal level at $8^{\text {th }}$ week of experiment as result of GSE intake in $1 \mathrm{~g} \mathrm{NaNO}_{2-}$ treated rats. Such enhancement in antioxidant profile by GSE intake against oxidative stress induced by many chemicals were previously recorded such as isoproterenol (Karthikeyan $\boldsymbol{e t}$ al., 2007), ciplatin (Yousef $\boldsymbol{e t}$ al., 2009 and Saad $\boldsymbol{e t}$ al., 2009) and streptozotocin (Chis $\boldsymbol{e t}$ al., 2009). 
Present histopathological results revealed that $\mathrm{NaNO}_{2}$ treatment for 8 weeks induced dose-dependent neurotoxicity. Brain of $1 \mathrm{~g} / \mathrm{L} \mathrm{NaNO}_{2}$-treated rats showed pyknosis of neurons, neuronophagia, menengial hemorrhage associated with inflammatory cells infiltration while brain of $2 \mathrm{~g} / \mathrm{L} \mathrm{NaNO}_{2}$-treated rats showed focal hemorrhage, hemorrhage in Virchow space, necrosis of neurons and congestion of cerebral blood vessels. $\mathrm{NaNO}_{2}$ induced neural toxic effects by exerting oxidative stress and retrograde the endogenous antioxidant system (Hassan $\boldsymbol{e t}$ al., 2010). Moreover hypoxic neurotoxicity was induced by $30 \mathrm{mg} / \mathrm{kg}$ of $\mathrm{NaNO}_{2}$ intake dissolved in normal water for 14 day and $75 \mathrm{mg} / \mathrm{kg}$ of subcutaneous injection $\mathrm{NaNO}_{2}$ (Premanand and Ganesh, 2010 and Zaidi, 2010, respectively).

Meanwhile, present study revealed that GSE intake significantly reversed neruotoxic effect of both $\mathrm{NaNO}_{2}$ dose which induced oxidative stress and restore brain tissue to normal. This suggestion run with data of Balu et al. (2005) demonstrated that GSE enhanced the antioxidant status and decreased the incidence of free radical induced protein oxidation in aged rats thereby protecting the central nervous system from the reactive oxygen species.

In the present investigation, treatment of rats by $\mathrm{NaNO}_{2}$ for 8 weeks resulted in lung injury as revealed by histological study. The degree of severity of pathological lesions was correlated to concentration of $\mathrm{NaNO}_{2}$. Microscopically, lungs of $1 \mathrm{~g} / \mathrm{L} \mathrm{NaNO}$-treatrd rats showed atelectasis and bronchitis. Whereas the examined lungs of rats treated with $2 \mathrm{~g} / \mathrm{L}$ $\mathrm{NaNO}_{2}$ showed perivascular inflammatory cells infiltration, interstitial pneumonia and pulmonary hemorrhage. Histopathologic changes in lung as a result of $\mathrm{NaNO}_{2}$ toxicity were also observed by Gruener and Shuval (1973) who stated that, the changes in the lungs consisted of dilatation of the bronchi with infiltration of lymphocytes and alveolar hyperinflation. U.S. EPA (1995) reported dilated bronchi, fibrosis and emphysema at 1000 ppm $\mathrm{NaNO}_{2}$ or above.

Present histopathological results revealed that GSE was able to protect lung against low $\mathrm{NaNO}_{2}$ dose and minimize damage of lung induced by high $\mathrm{NaNO}_{2}$ dose. The beneficial effect of GSE on lung tissue was previously reported by Hemmati et al (2008) revealed that the use of GSE can provide a protective action against the harmful and fibrogenic effect of silica on lung tissue.

Present histological examination revealed that $\mathrm{NaNO}_{2}$ induce dose-dependent testicular damage. Testes of rats administrated with $1 \mathrm{~g} / \mathrm{L} \mathrm{NaNO}{ }_{2}$ revealed degeneration of spermatogoneal cells lining seminiferous tubules and necrosis leydig cells. Also, examination of testes of rats treated with $2 \mathrm{~g} / \mathrm{L} \mathrm{NaNO}_{2}$ revealed interstitial edema and degeneration of spermatogoneal cells lining seminiferous tubules. The effect of $\mathrm{NaNO}_{2}$ on testis was previously reported by many studies. Akintunde et al. (2013) suggested that nitrite may change the testicular oxidative status and play a role in testicular dysfunction that causes infertility. Testes of rats received $0.04 \mathrm{mg} / \mathrm{kg} \mathrm{B.W}, 0.06 \mathrm{mg} / \mathrm{kg} \mathrm{B.W}$ and $0.08 \mathrm{mg} / \mathrm{kg}$ B.W nitrite by oral route for 30 days showed Seminiferous tubules with focal areas of lost germ cells, hyperplasia of Leydig cells and at $0.06 \mathrm{mg} / \mathrm{kg}$ dose level, there was arrest of spermatogenesis while there was no evidence of spermatogenesis at $0.08 \mathrm{mg} / \mathrm{kg}$ nitrite dose. Pavlova et al. (2013) reported that intraperitoneal injection of male Wistar rats with $\mathrm{NaNO}_{2}$ at dose of 50 mg.kg-1 B.W and sacrificed at different time intervals ( $1 \mathrm{~h}, 5 \mathrm{~h}, 24 \mathrm{~h}$ and $48 \mathrm{~h})$ following the administration resulted in disorganization of seminiferous epithelium and assemblance of undifferentiated germ cells in the luminal area of the tubules.

Present histopathological results revealed that GSE was able to protect testes against both $\mathrm{NaNO}_{2}$ dose and restore testicular tissue to normal. Such protective effect of GSE was recorded against sodium fluoride induced testicular damage (El-Demerdash et al., 2008).

Generally the cytotoxic and oxidative stress activity of $\mathrm{NaNO}_{2}$ may be due to $\mathrm{NO}$ and $\mathrm{ONOO}^{-}$formed from nitrite. Such product may increase lipid peroxidation which can be 
harmful to different organs (Chow and Hong, 2002). The high oxidative stress indicator lipid peroxidation could be attributed to the oxidative cytotoxicity of nitrite (Patsoukis and Georgiou, 2007). Moreover, nitrite can react with secondary and tertiary amines forming Nnitrosocompounds (nitrosamines) (Walker, 1990), which are associated with high risk of stomach, liver and esophagus carcinomas (Mitacek et al., 1999 and Kim et al., 2002). Nitrosamines have strong toxic, mutagenic, neuro- and nephrotoxic and carcinogenic effect. These may interpret the distortion in lung, testis, brain, heart, liver and kidney and reflected by the changes in the investigated parameters.

Meanwhile, GSE could reduce organ injury through its ability to balance the oxidantantioxidant status, and to regulate the release of inflammatory mediators (Sehirli et al., 2008). GSE has been shown to have conjugated structures between the B-ring catechol groups and $3-\mathrm{OH}$ free groups of polymeric skeleton allowing being effective free radical scavengers and metal chelators (Yilmaz and Toledo, 2004). As GSE scavenges free radicals, the resulting aroxyl radical formed has been shown to be more stable than that generated from other polyphenolics to prevent DNA damage (Bradham et al., 1998). Also, Devi et al. (2006) found that intake of proanthocyanidin which is a naturally occurring antioxidant from GSE in low quantity is effective in up-regulating the antioxidant defense mechanism by attenuating lipid peroxidation.

Conclusion: Sodium nitrite (preservative) has harmful effects on all tested parameters and induces oxidative damage in liver, kidney, brain, heart, lung and testis tissues in dosedependent manner. GSE not only has antioxidant properties that can enable it to scavenge the reactive oxygen species resulted from the ingestion of the food additives, but also it can normalize the changes induced by low $\mathrm{NaNO}_{2}$ dose in most examined organs. It has hypoglycemic and hypochlesterolemic actions.

\section{REFERENCES}

Abdul-Ameer, H.A. and Abed A.J. (2012): The Prophylactic Role of Garlic Oil against deleterious Effects of sodium nitrite $\left(\mathrm{NaNO}_{2}\right)$ in Male Mice. Al-Anbar J. Vet. Sci., 5(1):7-14.

Abebe, E.F.; Akpabio, C.J. and Maduagwu, E.N. (2013): Metabolism of precursors of Nnitrosamine in vitro and nitrosamine toxicology in wistar rat. Advances in Applied Sci. Res., 4(4):145-151.

Aboel-Zahab, H.; El-Khyat, Z.,; Sidhom, G., Awadallah, R.; Abdel-al, W. and Mahdy, K. (1997): Physiological effects of some synthetic food colouring additives on rats. Bollettino. Chimico. Farmaceutico., 136:615-627.

Akintunde, O.W.; Adefule, A.K., and Kehinde, B.D. (2013): Some adverse effects of nitrite on oxidative status and histological structures of adult male Wister rats testes. Am. J. Res. Communication, www.usa-journals.com.

Amico, V.; Napoli, E.M.; Renda, A.; Ruberto, G.; patafora, C. and Tringali, C. (2004): Constituents of grape pomace from the Sicilian cultivar "Nerello Mascalese". Food Chem., 88: 599-607.

Anandan, R.; Devi, K.P.; Devaki, T.and Govindaraju, P. (1998): Preventive effects of Picrorhiza kurroa on D-galatosamine-induced hepatitis in rats. Ind. J. Exp. Biol., $1,87-95$.

Anil, K.B.; Manju, B.; Giridhar, S. and Deepak, B. (2005): Protective role of Vitamin E pre-treatment on $\mathrm{N}$-nitrosodiethylamine induced oxidative stress in rat liver. Chem. Biol. Interact., 20:101-102. 
Bagchi, D.; Bagchi, M.; Stohs, S.J.; Das, D.K.; Ray, S.D.; Kuszynski, C.A.; Joshi, S.S. and Pruess, H.G. (2000): Free radicals and grape seed proanthocyanidin extract: Importance in human health and disease prevention. Toxicol., 148(2-3): 187-197.

Baky, N.A.A.; Zaidi, Z.F.; Fatani, A.J.; Sayed-Ahmed, M.M. and Yaqub, H. (2010): Nitric oxide pros and cons: The role of 1-arginine, a nitric oxide precursor, and idebenone, a coenzyme-Q analogue in ameliorating cerebral hypoxia in rat. Brain Res. Bull., 83:49-56.

Balu, M.; Sangeetha, P.; Murali, G. and Panneerselvam, C. (2005): Age-related oxidative protein damages in central nervous system of rats: modulatory role of grape seed extract. Int. J. Devl Neurosci., 23:501-507.

Banchroft, J.D.; Stevens, A. and Turner, D.R. (1996): Theory and Practice of Histological Techniques. Fourth (ed). Churchil Livingstone, NewYork, London, San Francisco, Tokyo, 125 pp.

Bentivegna, S.S. and Whitney, K.M. (2002): Subchronic 3-month oral toxicity study of grape seed and grape skin extracts. Food and Chemical Toxicol., 40:1731-1743.

Beutler, E., Duron, O. and Kelly, B.M. (1963): Improved method for the determination of blood glutathione. J. Lab Clin. Med.,61:882-888.

Bradham, C.A.; Plümpe, J.; Manns, M.P.; Brenner, D.A. and Trautwein, C.(1998): Mechanisms of hepatic toxicity. I. TNF-induced liver injury. Am. J. Physiol., 275:G387-392.

Carey, W.D. (2000): How should a patient with an isolated GGT be evaluated? Cleve. Clin. J. Med., 67: 315-316.

Chis, I.C.; Ungureanu, M.I.; Marton, A.; Simedrea, R.; Muresan, A.; Postescu, I. and Decea, N. (2009): Antioxidant effects of a grape seed extract in a rat model of diabetes mellitus. Diabetes \& Vascular Disease Res., 6(3): 200-204

Choi, S.Y.; Chung, M. J. and Sung, N. J. (2002): Volatile N-nitrosamine inhibition after intake of Korean green tea and Maesil (Prunus mume SIEB. ET ZACC.) extracts with an amine-rich diet in subjects ingesting nitrate. Food and Chemical Toxicol., 40: 949-957.

Chow, C.K. and Hong, C.B. (2002): Dietary vitamin E and selenium and toxicity of nitrite and nitrate. Toxicol.,180(2):195-207.

Cockburn, A.; Brambilla, G.; Fernández, M.L.; Arcella, D.; Bordajandi, L.R.; Cottrill, B.; van Peteghem, C. and Dorne, J.L. (2013): Nitrite in feed: from animal health to human health. Toxicol. Appl. Pharmacol., 270(3):209-17.

Craig, W. and Beck, L. (1999): Phytochemicals: Health Protective Effects. Can. J. Diet Pract. Res.; 60:78-84.

Cui, J.; Juhasz, B.; Tosaki, A.; Maulik, N. and Das, D.K. (2002): Cardioprotection with grapes. J Cardiovasc. Pharmacol., 40:762-769.

Delmas, D.; Jannin, B. and Latruffe, N. (2005): Resveratrol: preventing properties against vascular alterations and ageing. Mol. Nutr. Food Res.49: 377-395.

Dejam, A.; Hunter, C.J.; Pelletier, M.M.; Hsu, L.L.; Machado, R.F.; Shiva, S.; Power, G.G.; Kelm, M.; Gladwin, M.T. and Schechter, A.N. (2005): Erythrocytes are the major intravascular storage sites of nitrite in human blood. Blood, 106: 734-739. 
Devi, A.; Jolitha, A. B. and Ishii, N. (2006): Grape seed proanthocyanidin extract (GSPE) and antioxidant defense in the brain of adult rats. Med. Sci. Monit., 12(4):124-129.

Dulundu, E.; Ozel, Y.; Topaloglu, U.; Toklu, H.; Ercan, F.; Gedik, N. and Sener, G. (2007): Grape seed extract reduces oxidative stress and fibrosis in experimental biliary obstruction. J. Gastroenterol. Hepatol., 22(6):885-892.

EC (European Commission), (2002): Directive 2002/32/EC.. Council directive 2002/32/EC of 7 May 2002 on undesirable substances in animal feed (OJ L140, 30.5.2002, p.10).

Efuruibe, A.A.; Akpabio, C.J. and Maduagwu, E.N. (2013): Sub-cellular correlation of nitrite in cassava (Manihot EsculentaCrantz) leaves and nitrosamine toxicology in Wistar Rats. IJTPR, 5(3): 59-62.

El-Alfy, A.T.; Ahmed, A.A.E. and Fatani, A.J. (2005): Protective effect of red grape seeds proanthocyanidins against induction of diabetes by alloxan in rats. Pharmacol. Res. 52:264-270.

El-Beshbishy, H.A; Mohamadin, A.M.; Nagy, A.A. and Abdel-Naim, A.B. (2010): Amelioration of tamoxifen-induced liver injury in rats by grape seed extract, black seed extract and curcumin. Indian J. Exp. Biol., 48(3):280-288.

El-Demerdash, M.; Abd El khalik,A.M.; Abou El Magd, S.A. and Gharib, A.F. (2008): Possible protective role of grape seed proanthocyanidin on fluoride induced testicular toxicity in adult and young albino rats. Mansoura J. Forensic Med. Clin. Toxicol., XVI(1): 98-107.

El-Sheikh, N.M. and Khalil, F.A. (2011): L-Arginine and L-glutamine as immunonutrients and modulating agents for oxidative stress and toxicity induced by sodium nitrite in rats. Food Chemical Toxicol., 49:758-762

Eremin, Y.N. and Yocharina, M.G. (1981): Effect of nitrites on the state of thyroid gland in iodine deficiency and different diets. Vopr. Pitan. 5:60-62.

Filvo, V.A., Ivin, B.A., Bandman, A.L. (Eds.), (1993): Harmful Chemical Substances: Elements in Group IIV of the Periodic Table and Their Inorganic Compounds. Ellis Horwood, Prertic Hall, West New York. p. 722.

Gladwin, M.T.; Crawford, J.H. and Patel, R.P. (2004): The biochemistry of nitric oxide, nitrite, and hemoglobin: role in blood flow regulation. Free Radical Biology and Medicine. 36, 707-717.

Gluhcheva, Y.; Ivanov, I.; Petrova, E.; Pavlova, E.; Vladov, I. (2012): Sodium nitriteinduced hematological and hemorheological changes in rats. Series on Biomechanics, 27(3-4):53-58.

Gomaa, G.M.; Abd Elaziz, E.A. (2011): Protective effect of aquaeous green tea extract on toxicity caused by sodium nitrite. Assiut Vet. Med. J., 57:138-162.

Gruener, N. and Shuval, H. (1973): Health Implications of Environmental Exposure to Nitrates. Harefuah. 85:30-32.

Habig, W.H.; Pabst, M.J. and Jakoby, W.B. (1974): Glutathione S-transferases. The first enzymatic step in mercapturic acid formation. J. Biol. Chem., 25;249(22):71307139. 
Hassan H. A.; El-Agmy, S.M.; Gaur, R.L.; Fernando, A.; Raj, M.H.G. and Ouhtit, A. (2009): In vivo evidence of hepato- and reno-protective effect of garlic oil against sodium nitrite-induced oxidative stress. Int. J. Biol. Sci., 5:249-255.

Hassan, H.A., Hafez, H.S. and Zeghebar, F.E. (2010): Garlic oil as a modulating agent for oxidative stress and neurotoxicity induced by sodium nitrite in male albino rats. Food and Chemical Toxicol., 48:1980-1985.

Hassan, H.M.M. (2012): Hepatoprotective effect of red grape seed extracts against ethanolinduced cytotoxicity. Global J. Biotech. \& Biochem., 7 (2): 30-37.

Helal, E.G.E. and Elsaid, F.G.G. (2006): Management the action of sodium nitrite on albino rats by aqueous garlic extract. Res. J. Medicine \& Med. Sci., 1(3): 85-89.

Hemmati, A.A.; Nazari, Z. and Samei, M. (2008): A comparative study of grape seed extract and vitamin $\mathrm{E}$ effects on silica-induced pulmonary fibrosis in rats Pulmonary. Pharmacol. Therapeutics, 21:668-674.

Hirneth, H. and Classen, H.G. (1984): Inhibition of nitrate-induced increase of plasma nitrite and methemoglobin in rats by simultaneous feeding of ascorbic acid or tocopherol. Arzneimittelforschung, 34(II): 988-991.

Imaizumi, K.; Tyuma, I.; Imai, K.; Kosaka, H. and Ueda, Y. (1980): In vivo studies on methemoglobin formation by sodium nitrite. International Archives of Occupational and Environmental Health, 45:97-104.

Jahries, G.; Hesse, V.I.; Schone, L.H. and Mehnert, E. (1986): Influence of nitrates and plant goitrgens on thyroid hormone, somated in status and growth of swine. J. Vet. Med., 41: 528-530.

Jensen, F.B. (2007): Nitric oxide formation from nitrite in Zebra fish. J. Exp. Biol. 210:3387-3394.

Jiao, R.; Zhang, Z.; Yu, H.; Huang, Y. and Chen, Z. (2010): Hypocholesterolemic activity of grape seed proanthocyanidin is mediated by enhancement of bile acid excretion and up-regulation of CYP7A1. J. Nutr. Biochem., 21:1134-1139.

Karthikeyan, K.; Sarala Bai, B.R.; Devaraj S.N. (2007): Efficacy of grape seed proanthocyanidins on serum and heart tissue lipids in rats subjected to isoproterenol-induced myocardial injury. Vasc. Pharmacol. 47:295-301.

Kaya, K. and Miura, T. (1982): Selective changes in fatty acid composition of phosphatidylserine in rat erythrocyte membrane induced by nitrate. Biochimica. et Biophysica. Acta., 688:305-315.

Kim, H.J.; Chang, W.K.; Kim, M.K.; Lee S.S. and Choi, B.Y. (2002): Dietary factors and gastric cancer in Korea: Case-control study. Int. J. Cancer, 97: 531-535.

Kohn, M.C.; Melnick, R.L.; Ye, F. and Portier, C.J. (2002): Pharmacokinetics of sodium nitrite induced methemoglobinemia in the rat. Drug Metabolism and Disposition, 30:676-683.

Kolpakov, V.; Gordon, D. and Kulik, T.J. (1995): Nitric oxide-generating compounds inhibit total protein and collagen synthesis in cultured vascular smooth muscle cells. Circ. Res., 76:305-309.

Krishnamoorthy,P. and Sangeetha, M. (2008): Hepatoprotective effect of vitamin C on sodium nitrite-induced lipid peroxidation in albino rats. IJBB,45(3), 206-208. 
Lai, L.S.; Chou, S.T. and Chao, W.W. (2001): Studies on the antioxidative activities of Hsian-tsao (Mesonaprocumbens Hemsl) leaf gum. J. Agric. Food Chem. 49: 963968.

Lane-Peter, W. and Pearson, A.E.G. (1971): Dietary requirement cited in "The laboratory animal principles and practice". $1^{\text {st }}$ Academic press, London and New York. 142.

Levey, A.S.; Bosh, J.P.; Lewis, J.B.; Green, T.; Rogers, N. and Roth, D. (1999): A more accurate method to estimate glomerular filtration rate from serum crearinine: a new prediction equation. Modification of diet in renal disease study group. Ann. Int. Med. 130: 461-470.

Liaudet, L; Andrea, S.; Garcia, S.F.; Basilia, Z.; Csaba, S. and Andrew, S.L. (2000): Poly (Adp-Ribose) synthetase mediates intestinal mucosal barrier dysfunction after mesenteric ischemia. Shock, 14 (2):134-141.

Luca, V.; Cotor, F. and Raileanu, L. (1987): In vivo and in vitro cytogenetic damage induced by sodium nitrite. Mutat. Res. 189, 333-339.

Lundberg, J.O.; Weitzberg, E.; Lundberg, J.M. and Alving, K. (1994): Intragastric nitric oxide production in humans: measurements in expelled air. Gut., 35(11):15431546.

Maeda, N.; Imaizumi, K.; Kon, K. and Shiga, T. (1987): A kinetic study on functional impairment of nitric oxide-exposed rat erythrocytes. Environ. Health Perspectives, 73: 171-177.

McKnight, G.M.; Smith, L.M.; Drummond, R.S.; Duncan, C.W.; Golden, M. and Benjamin, N. (1997): Chemical synthesis of nitric oxide in the stomach from dietary nitrate in humans. Gut., 40: 211-214.

Mirvish, S.S.; Greenblatt, M. and Choudari Kommineni, V. R. (1972): Nitrosamide formation in vivo: Induction of lung adenomas in Swiss Mice by concurrent feeding of nitrite and methylurea or ethylurea. J. Nat. Canc. Inst., 48(5), 13111315.

Mitacek, E.J.; Brunnemann, K.D.; Suttajit, M.; Martin, N.; Limsila, T.; Ohshima, H.and Caplan, L.S. (1999): Exposure to N-nitrosocompounds in a population of high liver cancer regions in Thailand: Volatile nitrosamine (VNA) levels in Thai food. Food Chemical Toxicol., 37: 297-305.

Mukherjee, S.; Banerjee, S.K.; Maulik, M.; Dinda, A.K.; Talwar, K.K. and Maulik, S.K. (2003): Protection against adriamycin-induced cardiotoxicity by garlic: role of endogenous antioxidants and inhibition of TNF-a expression. BMC Pharmacol. $3: 1-9$.

Nishikimi, M.; Rao, N.A. and Yagi, K. (1972): The occurrence of superoxide anion in the reaction of reduced phenazinemethosulfate and molecular oxygen. Biochem. Biophys. Res. Commun., 46: 849-854.

Onkawa, H.; Ohishi, N.; Yagi, K. (1979): Assay for lipid peroxides in animal tissues by thiobarbituric acid reaction. Anal. Biochem., 95:351-358.

Patsoukis, N. and Georgiou, C.D. (2007): Effect of sulfite-hydrosulfite and nitrite on thiol redox state, oxidative stress and sclerotial differentiation of filamentous phytopathogenic fungi. Pestic. Biochem. Physiol. 88:226-235. 
Pavlova, E.; Dimova, D.; Petrova, E.; Gluhcheva, Y. and Atanassova, N. (2013): Changes in rat testis and sperm count after acute treatment with sodium nitrite. Bulg. J. Agric. Sci., Supplement 2, 19: 186-189.

Pinent, M.; Blay, M.; Blade, M.; Salvado, M.; Arola, L. and Ardevol, A. (2004): Grape seed-derived procyanidins have an antihyperglycemic effect in streptozotocininduced diabetic rats and insulinomimetic activity in insulin-sensitive cell lines. Endocrinol., 145:4985-4990.

Premanand, R. and Ganesh, T. (2010): Neuroprotective effects of Abrus precatorius Linn. aerial extract on hypoxic neurotoxicity induced rats. Int. J. Chemical Pharm. Sci., $1(1): 9-15$.

Radi, R.; Beckman, J.S.; Bush, K.M. and Freeman, B.A. (1991): Peroxynitrite oxidation of sulfhydryls. The cytotoxic potential of superoxide and nitric oxide. J. Biol. Chem., 266(7):4244-4250.

Raj, K.J. and Shalini, K. (1999): Flavonoids - a review of biological activities. Indian Drugs; 36:668-676.

Rao, P. and Bhat, R.V . (2003): A comparative study on the synthetic food colours usage in foods procured from urban and rural areas of Hyderabad. Nutr. Food Sci., 33: 230234.

RCHAS (Reproductive and Cancer Hazard Assessment Section) (2000): Office of Environmental Health Hazard Assessment (OEHHA), California Environmental Protection Agency (CAL/EPA): Evidence on development and reproductive toxicity of sodium nitrite.

Ricardo-DaSilva, J.M.; Darmon, N.; Fernandez, Y. and Mitjavila, S. (1991): Oxygen free radical scavenger capacity in aqueous models of different procyanidins from grape seeds. J. Agri. Food Chem., 39 (9): 1549-1552.

Roychowdhury, S.; Wolf, G.; Keilhoff, G.; Bagchi, D. and Horn, T. (2001): Grape seed proanthocyanidin extract (GSPE) protects astroglia against nitrosative/oxidative stress. Nitric Oxide, 5: 137-149.

Saad, A.A.; Youssef, M.I. and El-Shennawy, L.K. (2009): Cisplatin induced damage in kidney genomic DNA and nephrotoxicity in male rats: The protective effect of grape seed proanthocyanidin extract. Food and Chemical Toxicol., 47:1499-1506.

Saito, M.; Hosoyama, H.; Ariga, T.; Kataoka, S. and Yamaji, N. (1998): Antiulcer activity of grape seed extract and procyanidins. J. Agric. Food Chem., 40: 1460-1464.

Salter, A.M. and White, D.A. (1996): Effects of dietary fat on cholesterol metabolism: regulation of plasma LDL concentrations. Nutr. Res., 9:241-257.

Sanchez-Echaniz, J.; Benito-Frnandez, J. and Mintegui-Raso, S. (2001): Methemoglobinemia and consumption of vegetables in infants. Pediatrics, 107: 1024-1028.

Santos-Buelga, C.; Francia-Aricha, E.M. and Escribano-Bailón, M.T. (1995): Comparative flavan-3-ol composition of seeds from different grape varieties. Food Chem., 53: 197-20.

Sato, S.; Hori, Y.; Yamate, J.; Saito, T.; Kurasaki, M. and Hatai, A. (2005): Protective effect of dietry azuki bean (Vigna angularis) seed coats against renal interstitial fibrosis of rats induced by cisplatin. Nutr. 21:504-511. 
Sehirli, O.; Ozel, Y.; Dulundu, E.; Topaloglu, U.; Ercan, F. and Sener, G. (2008): Grape seed extract treatment reduces hepatic ischemia-reperfusion injury in rats. Phytother. Res.,22(1):43-48.

Shanmuganayagam, D.; Beahm, M.R.; Osman, H.E.; Krueger, C.G.; Reed, J.D. and Folts, J.D. (2002): Grape seed and grape skin extracts elicit a greater antiplatelet effect when used in combination than when used individually in dogs and humans. J. Nutr., 132:3592-3598.

Shi, J.; Yu, J.; Pohorly, J.E. and Kakuda Y. (2003): Polyphenolics in grape seedsbiochemistry and functionality. J. Med. Food., 6(4):291-299.

Snedecor, G.W. and Cochran, W.G. (1980): Statistical methods. $7^{\text {th }}$ (ed). Iowa state University Press, Ames, Iowa, USA.

Subramaniyan, N.K.; Adimoolam, S.; Paramasivam, S.K.; Sulaiman, M. and Delhiraj, $\boldsymbol{H}$. (2011): Free radical scavenging activity of evolvulus alsinoides on hypoxia induced neurodegenration. IJRAP, 2(1):302-305.

Tan, Y.S.; Nambiar, R. and Yo, S.L. (1992): Prevalence of protein calorie malnutrition in general surgical patients. Annals of the Academy of Medicine, Singapore, 21: 334- 338 .

Terra, X.; Montagut, G.; Bustos, M.; Llopiz, N.; Ardèvol, A.; Bladé, C.; FernándezLarrea,J.; Pujadas, G.; Salvadó, J.; Arola, L. and Blay, M. (2009): Grape-seed procyanidins prevent low-grade inflammation by modulating cytokine expression in rats fed a high-fat diet. J. of Nutr. Biochem., 20: 210-218.

Torres, J.L.; Varela, B.; García, M.T.; Carilla, J.; Matito, C.; Centelles, J.J.; Cascante, M.; Sort, X. and Bobet, R. (2002): Valorization of grape (Vitis vinifera) byproducts. Antioxidant and biological properties of polyphenolic fractions differing in procyanidin composition and flavonol content. J. Agric. Food Chem., 50: $7548-7555$.

U.S. EPA (1995). Integrated Risk Information Service (IRIS), 1995 data base. Last revised 1991.

Valavanidisa, A.; Vlahogiannia, T.; Dassenakisb, M. and Scoullosb, M. (2006): Molecular biomarkers of oxidative stress in aquatic organisms in relation to toxic environmental pollutants. Ecotoxicol. Environ. Saf. 64:178-189.

Walker, R. (1990): Nitrates, nitrites and N-nitroso compounds: A review of the occurrence in food and diet and the toxicological implications. Food additives and Contaminants, 7: 717-768.

Waterhouse, A.L. and Walzem, R.L. (1998): Nutrition of Grape Phenolics. In Flavonoids in Health and Disease; Rice-Evans, C., Packer, L., Eds.; Marcel Dekker, Inc.: New York, NY,; pp. 349-387.

WHO (World Health Organization) (2003): Safety evaluation of certain food additives. Fifty-fourth Report of the Joint FAO/WHO Committee on Food Additives.Food Additives Series No. 50.Genera: WHO.

WHO (World Health Organization) (2007): Nitrate and nitrite in drinking water. Background document for development of WHO Guidelines for Drinking-water Quality. 
Wiechetek, M.; Garwacki, S.; Karlik, W.; Lewicki, J. and Souffrant, W. (1992): Effect of nitrite on ureagenesis and carbohydrate metabolism in isolated rat hepatocytes. Arch. of Environ. Contamin. Toxicol., 24:375-380.

Wolff, I.A. and Wasserman, A.E. (1972): Nitrates, nitrites, and nitrosamines. Sci., 177(4043):15-9.

Wren, A. F.; Cleary, M.; Frantz, C.; Melton, S. and Norris, L.(2002): 90-Day Oral Toxicity Study of a Grape Seed Extract (IH636) in Rats. J. Agric. Food Chem., 50(7):2180-2192.

Yilmaz, Y.;Toledo, R.T.(2004): Health aspects of functional grape seed constituents. Trends in Food Sci. Technol., 15 (9):422-433.

Yousef, M.I; Saad, A.A. and El-Shennawy, L.K. (2009): Protective effect of grape seed proanthocyanidin extract against oxidative stress induced by cisplatin in rats. Food and Chemical Toxicol., 47:1176-1183.

Zaidi, Z.F. (2010): Effect of sodium nitrite-induced hypoxia on cerebellar purkinje cells in adult rats. Pak. J. Med. Sci., 26 (2): 261-266.

Zatar, N.A.; Abu-Eid M. and Eid, A.F. (1999): Spectrophotometric determination of nitrite and nitrate using phosphomolybdenum blue complex. Talanta, 50: 819-826.

Zurovsky, Y. and Haber, C., (1995): Antioxidants attenuate endotoxin-generation induced acute failure in rats. Scand. J. Urol. Nephrol. 29:147-154.

$$
\begin{aligned}
& \text { التأثير الوقائى لمستخلص بذور العنب ضد السمية و الجهر المؤكسد الناتج عن نتريت الصوديوم فى الجى } \\
& \text { الجرذان الئه } \\
& \text { "جيهان مصطقى حمود، نيفين سعد نايل، خالد محما عبد الرحيم الصاوى ، عادل عادل سالم عبد الرحمن ، مرفت سيد حسن }
\end{aligned}
$$

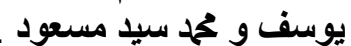

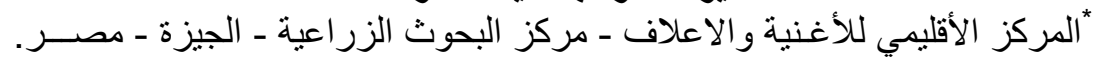

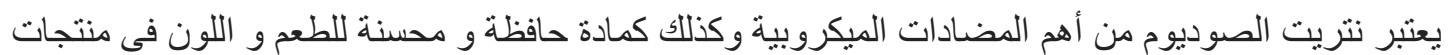

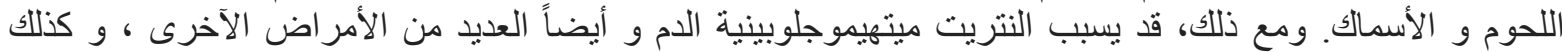

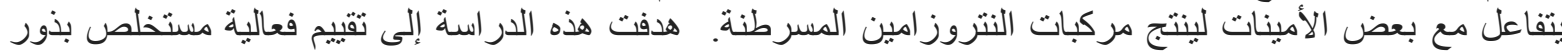

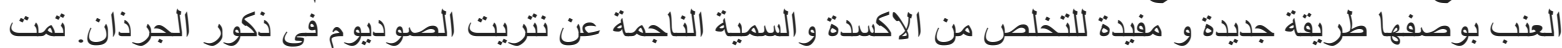

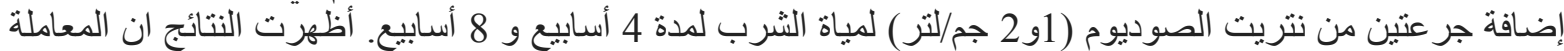

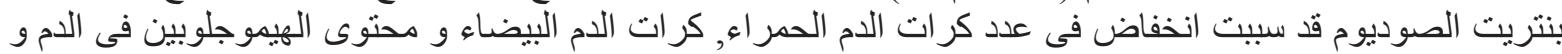

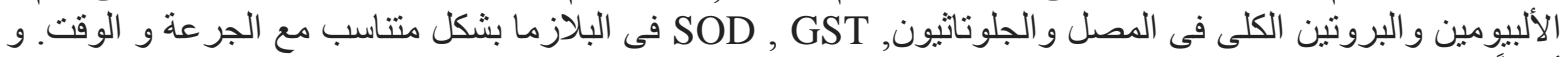

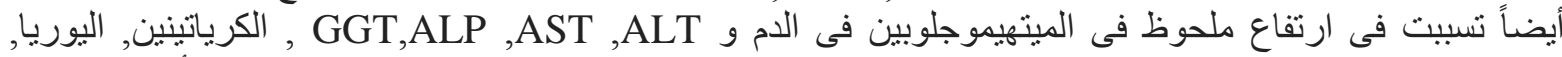

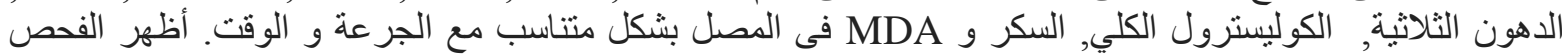

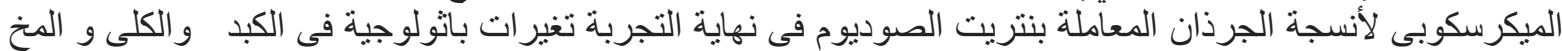

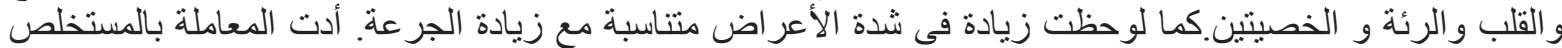

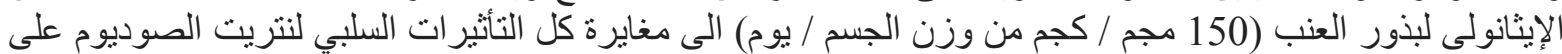

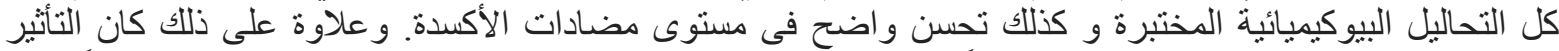

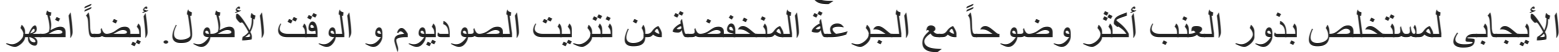

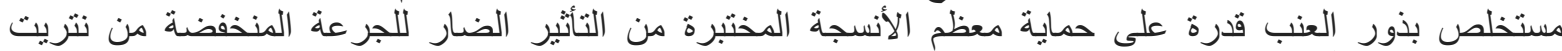

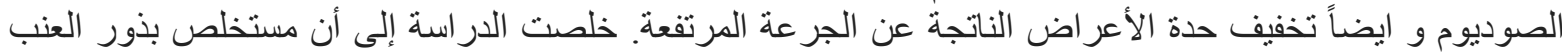

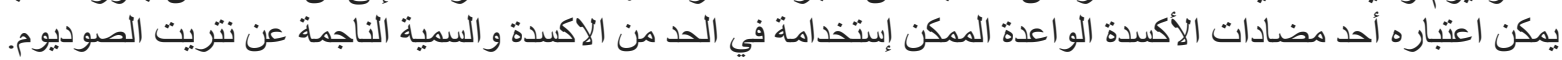
الكلمات الدالة: نتريت الصوديوح, مستخلص بذور العنب, الجهد المؤكسد, التحاليل البيوكيميائية و الفحص النسيجى. 